\title{
3-D Vector Flow Estimation With Row-Column-Addressed Arrays
}

Holbek, Simon; Christiansen, Thomas Lehrmann; Stuart, Matthias Bo; Beers, Christopher; Thomsen, Erik Vilain; Jensen, Jørgen Arendt

Published in:

I E E E Transactions on Ultrasonics, Ferroelectrics and Frequency Control

Link to article, DOI:

10.1109/TUFFC.2016.2582536

Publication date:

2016

Document Version

Peer reviewed version

Link back to DTU Orbit

Citation (APA):

Holbek, S., Christiansen, T. L., Stuart, M. B., Beers, C., Thomsen, E. V., \& Jensen, J. A. (2016). 3-D Vector Flow Estimation With Row-Column-Addressed Arrays. I E E E Transactions on Ultrasonics, Ferroelectrics and Frequency Control, 63(11), 1799-1814. https://doi.org/10.1109/TUFFC.2016.2582536

\section{General rights}

Copyright and moral rights for the publications made accessible in the public portal are retained by the authors and/or other copyright owners and it is a condition of accessing publications that users recognise and abide by the legal requirements associated with these rights.

- Users may download and print one copy of any publication from the public portal for the purpose of private study or research.

- You may not further distribute the material or use it for any profit-making activity or commercial gain

- You may freely distribute the URL identifying the publication in the public portal 


\title{
3-D Vector Flow Estimation with Row-Column Addressed Arrays
}

\author{
Simon Holbek, Thomas Lehrmann Christiansen, Matthias Bo Stuart, Christopher Beers, Erik Vilain Thomsen, and \\ Jørgen Arendt Jensen
}

\begin{abstract}
Simulation and experimental results from 3-D vector flow estimations for a 62+62 2-D row-column (RC) array with integrated apodization are presented. A method for implementing a 3-D transverse oscillation (TO) velocity estimator on a 3.0 MHz RC array is developed and validated. First, a parametric simulation study is conducted where flow direction, ensemble length, number of pulse cycles, steering angles, transmit/receive apodization, and TO apodization profiles and spacing are varied, to find the optimal parameter configuration. The performance of the estimator is evaluated with respect to relative mean bias $\tilde{B}$ and mean standard deviation $\tilde{\sigma}$. Second, the optimal parameter configuration is implemented on the prototype $\mathrm{RC}$ probe connected to the experimental ultrasound scanner SARUS. Results from measurements conducted in a flow-rig system containing a constant laminar flow and a straight-vessel phantom with a pulsating flow are presented. Both an M-mode and a steered transmit sequence are applied. Three-dimensional vector flow is estimated in the flow-rig for four representative flow directions. In the setup with $90^{\circ}$ beam-to-flow angle, the relative mean bias across the entire velocity profile is $(-4.7,-0.9,0.4) \%$ with a relative standard deviation of $(8.7,5.1,0.8) \%$ for $\left(v_{x}, v_{y}, v_{z}\right)$. The estimated peak velocity is $48.5 \mathrm{~cm} / \mathrm{s} \pm 3.0 \mathrm{~cm} / \mathrm{s}$ giving a $-3 \%$ bias. The out-of-plane velocity component perpendicular to the cross section is used to estimate volumetric flow rates in the flow-rig at a $90^{\circ}$ beam-to-flow angle. The estimated mean flow rate in this setup is $91.2 \mathrm{~L} / \mathrm{h} \pm 3.1 \mathrm{~L} / \mathrm{h}$ corresponding to a bias of $\mathbf{- 1 1 . 1 \%}$. In a pulsating flow setup, flow rate measured during five cycles is $2.3 \mathrm{~mL} / \mathrm{stroke} \pm \mathbf{0 . 1} \mathrm{mL} / \mathrm{stroke}$ giving a negative $9.7 \%$ bias. It is concluded that accurate 3-D vector flow estimation can be obtained using a 2-D RC addressed array.
\end{abstract}

\section{INTRODUCTION}

Real-time estimation of blood velocities is a valuable tool for diagnosing cardiovascular diseases [1]. Currently, most commercial scanners are only able to estimate the axial velocity component, which is used for diagnosing the degree of stenosis and for volume flow estimation [1]-[4]. The technique is subject to the angle-dependency problem, which leads to inaccuracies in the estimates [5], [6]. This problem was solved with 2-D vector flow techniques as speckle tracking [7], [8], directional beamforming [9], [10], vector Doppler techniques [8], [11]-[14] and the transverse oscillation (TO) method [15][17]. However, since blood propagates in all three dimensions, the true dynamics are only revealed with $3-\mathrm{D}$ vector flow.

This work was financially supported by grants 82-2012-4 from the Danish National Advanced Technology Foundation and from BK Ultrasound ApS (Herlev, Denmark).

S. Holbek, M.B. Stuart and J.A. Jensen are with the Center for Fast Ultrasound Imaging, Department of Electrical Engineering, Technical University of Denmark, Kgs. Lyngby, Denmark.

T.L. Christiansen and E.V. Thomsen are with the Department of Micro- and Nanotechnology, Technical University of Denmark, Kgs. Lyngby, Denmark.

C. Beers is with Sound Technology, Inc., State College, Pennsylvania, USA
Several techniques have been proposed for 3-D velocity estimation using either a 1-D transducer [18], [19] or a 2-D matrix array [20]-[22]. The drawback of the proposed methods with a 1-D transducer is that they often are not providing real-time estimates or the setup and calibration scheme are very cumbersome. Although good results have been presented in the literature with a 2-D matrix array, this type of probe faces various fabrication issues and processing limitations. One major drawback with the matrix probe is that the total number of interconnections in a $N \times N$ element transducer, scales with $N^{2}$, which causes connection issues. Moreover, the generated amount of channel data complicates the task of real-time processing. Thus, solutions for 3-D imaging with low channel count 2-D transducers have been investigated in the literature.

One of the ideas that has emerged, is to create a 2-D rowcolumn (RC) addressed array [23], [24]. A RC addressed array can be viewed as two orthogonally oriented quadratic 1-D arrays with tall elements, mounted on top of each other. Compared to an $N \times N$ matrix array, the total number of interconnections in an $N+N \mathrm{RC}$ array is reduced by a factor of $N / 2$, which eases the interconnect and opens up for transducers with both a large footprint and a small pitch.

Several versions and layouts of 2-D RC arrays have been presented for imaging purposes, for both piezoelectric arrays [25], [26] and Capacitive Micromachined Ultrasonic Transducers (CMUTs) [27]-[29]. However, the potential of estimating blood flow with RC arrays has only recently been reported in the literature [30]. Due to the low channel count on such an array, a realtime implementation of 3-D vector flow estimation in the clinic seems highly feasible.

This work develops a technique for 3-D vector flow estimation on a 2-D $62+62$ piezo RC prototype probe with integrated element apodization, using the TO method. The RC probe was made in collaboration with Sound Technology, Inc. (State College, PA, USA) and has similar parameters as the $62+62 \mathrm{RC}$ CMUT probe presented in previous work for experimental use [29]. The feasibility of the technique is explored in a simulation study, where several parameters are varied, including beam steering angle, flow angle, ensemble lengths and apodization profiles, to optimize the setup. The conclusions drawn from the parametric study are used to optimize the experimental setup using the prototype probe. Experiments with the RC probe were used to demonstrate the feasibility of estimating 3-D vector flow both in a flow-rig, containing a laminar parabolic flow profile, and in a vessel where a flow pump was driving a pulsatile carotid waveform. 


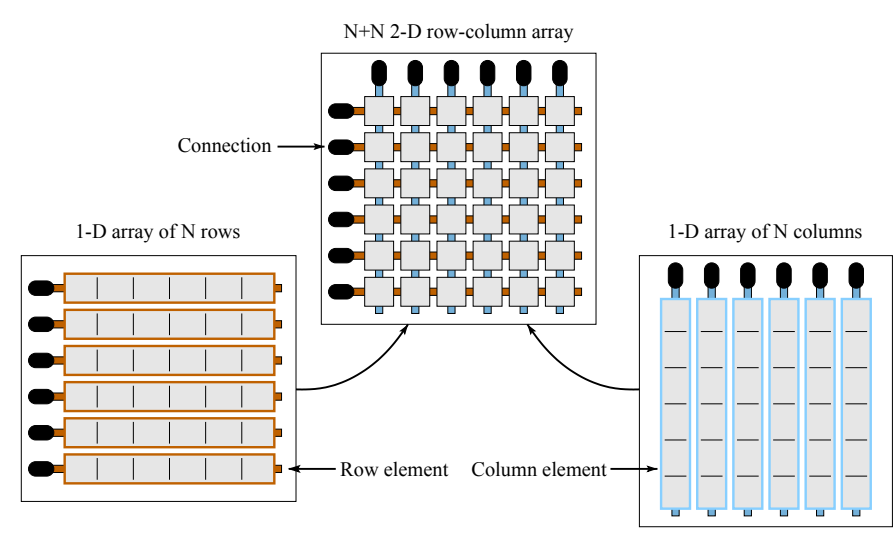

Figure 1: Two $1 \times N$ and $N \times 1$ 1-D one-way focused linear arrays are mounted orthogonally on top of each other to form the 2-D row-column addressed $N+N$ array. Modified from [31].

The paper expands on previous work [30]. Specifically, the simulations part has been extended from one single parameter setup to cover a full parametric study, and the experimental setup has been expanded to cover intensity measurements, different flow directions, a steered transmit sequence, and measurements on pulsatile flow.

\section{2-D ROW-COLUMN ADDRESSED ARRAYS}

When describing a 2-D RC addressed array as two orthogonally oriented quadratic 1-D arrays on top of each other, it seems valid to apply current theories and techniques on these transducer arrays. However, when working with 2-D $\mathrm{RC}$ arrays, several precautions are necessary regarding e.g. conventional approximations about transducer geometry as well as some restrictions that are present in both transmit and receive. This section covers the most common precautions and how they were resolved in this work.

\section{A. The concept of $R C$}

The concept of a 2-D RC addressed array is easiest described as a $1 \times N$ and a $N \times 1$-D array merged together to form a $N+N$ array, which can be accessed from either its row index or column index as seen in Fig. 1. In transmit, any number or combination of elements can be excited at a time, as long as they are oriented in the same direction. In receive, however, data from all $2 N$ elements can be accessed simultaneously [29]. Focusing is performed in one lateral dimension by electronically applying a time delay to each element, as described in Section II-D.

A 2-D RC array can be viewed as a special case of a 2-D matrix array, where whole lines of elements are connected to work as a unit. The reduced flexibility in transmit, and the lack of access to element data from $N^{2}$ channels with a RC array, translates to reduced fabrication complexity and data processing requirements, which eases a real-time implementation.

\section{B. Integrated apodization}

Despite the advantages that follows with the RC arrays, one inherent drawback comes from its tall elements, which produces several ghost echoes emerging from the element edges [24], [31]. The largest amplitude of the ghost echo is approximately $40 \mathrm{~dB}$ lower than the amplitude of the main echo. The scattered signal from a blood cell is typical -40 $\mathrm{dB}$ less than the echo from the surrounding vessel wall, which stresses the importance of suppressing these artifacts for accurate velocity estimation. It was shown that edge waves could be reduced without affecting the main echo by implementing a roll-off apodization at the end of each line element [32]. Based on these suggestions, each end of the line elements include a Hann shaped roll-off apodization of length $16 \times$ pitch.

\section{Beamforming RC data}

Conventional delay-and-sum beamforming assumes that the geometry of transmitting and receiving elements are point sources emitting spherical waves. The emitted wavefront from a single RC element is, however, shaped as a cylindrical surface, where it can be viewed as a circle arc in a plane orthogonal to the long dimension and as a plane wave in the plane orthogonal to the short dimension. Therefore, the point source approximation breaks down even for small RC arrays and leads to errors in the time-of-flight calculations. A better approximation treats the elements as line segments, which influences the time-of-flight calculations used in the delay-and-sum beamformer [32].

The applied beamformer [32] takes the position of the emitting element (source), the time of emission, and the position of the receiving elements (drains) as input. When beamforming data from focused emissions, where multiple elements are emitting according to a specified delay curve the same approach as for single element emissions was used. Thus, the element with the smallest delay time was chosen as the source, and all elements in the orthogonal dimensions were used as drains in the beamformer.

\section{Focusing with RC arrays}

As previously stated, the wavefront from a single RC element can be viewed as a plane wave in one plane and a circle arc in the orthogonal plane. Therefore, when multiple line elements are excited according to a specified delay curve, the wavefronts will add up to form a focal line rather than a focal point. Steered electronically focused emissions are possible, but only in the direction orthogonal to the transmitting line elements. The focal line spans the dimension of the excited elements as seen in Fig. 2, where the maximum pressure for a focused emission at the focal depth is simulated.

If the same elements are used for both transmitting and receiving, the beamformed data will correspond to the averaged received echo along the focal line, since focused emissions generate a line shaped pressure field. Due to this, beamforming was made with elements oriented orthogonally to the emitting elements. These issues with RC arrays have to be taken into consideration when designing the emission sequence. 


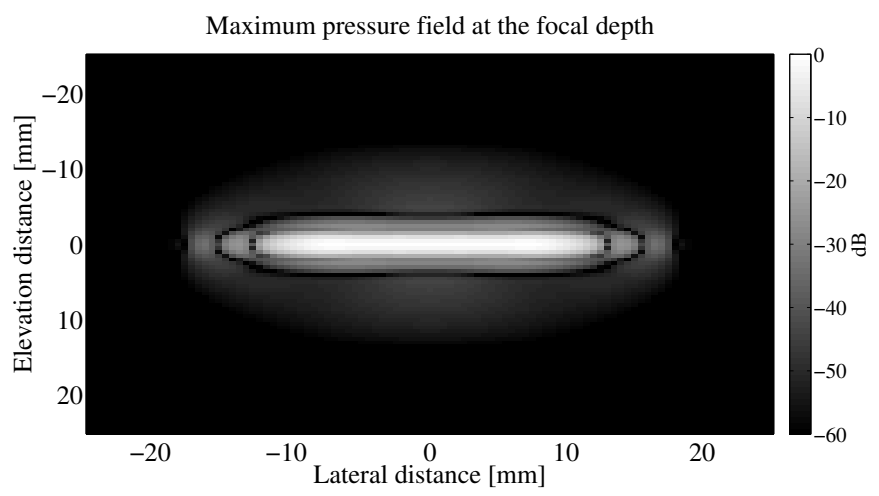

Figure 2: Pressure field generated with focused emissions. A focal line rather than a focal point is made with a RC array. The simulation parameters were the default values described in Table I.

\section{THE TRANSVERSE OSCILLATION METHOD}

Several approaches and applications of TO have been reported in the literature, for instance for tissue displacement estimation [33]-[35] and for vector flow estimation [15]-[17], [20]. This work explores a TO method for estimating vector flow by generating two decoupled double-oscillating fields in receive. The received signals are affected by axial or transverse blood flow motion, which propagates through the doubleoscillating fields. By using a phase-shift estimator, the axial and transverse motion can be detected. The basic steps of the applied TO method are described in the following section. A more thorough explanation of the theory is given in [15], [16], [20].

\section{A. Basic concept}

The idea of the TO method is to develop an angleindependent 2-D velocity estimator by generating two double oscillating fields (left and right) in receive. Ideally, the right field should be a spatially Hilbert transformed version of the left one. Spatial quadrature sampling is needed to estimate the axial and transverse velocity components and their direction.

The two $90^{\circ}$ shifted double-oscillating fields are made by a spatial transverse separation of $\lambda_{x} / 4$ in the beamforming, where $\lambda_{x}$ is the transverse oscillation period. The oscillation period is controllable and determined by the receive apodization profile. The apodization should contain two symmetrically placed peaks separated by the distance $d_{x}$. At a certain axial depth $z, \lambda_{x}$ is calculated as

$$
\lambda_{x}(z)=2 \lambda_{z} \frac{z}{d_{x}}
$$

where $\lambda_{z}$ is the wavelength of the emitted pulse in the medium. Being able to control the transverse wavelength by changing the distance between the two apodization peaks is one of the advantages of the method. As the maximum detectable velocity scales with $\lambda_{x}$, it provides a flexible tool for estimating both low and high velocities.

\section{B. Beamforming}

With the applied TO method, it is possible to estimate two velocity components, if a 1-D array is used; one component perpendicular to the element orientation in addition to the axial component. Three beamformed lines are needed for this; one center line for the axial estimator and two steered lines for the transverse estimate. The center line $r_{\text {center }}$ is beamformed along the direction $(0,0, z)$, using delay-and-sum and a traditional apodization profile. For the two steered lines, a traditional TO apodization profile with two separated peaks is applied and beamforming is performed along the lines $(x$, $y, z)=\left( \pm \lambda_{x}(z) / 8,0, z\right)$ to create the $\lambda_{x} / 4$ spatial separation.

The method can be expanded to estimate $3-\mathrm{D}$ velocities, if a 2-D array is used. The third velocity component can be obtained by applying the same procedure as for the transverse component, but this time by beamforming the two steered lines at $\pm \lambda_{y} / 8$ in the orthogonal direction. All five lines can either be beamformed in parallel from the same transmission or be combined from multiple consecutive transmission. For RC TO, the last approach was used, for reasons explained in Section IV. The five beamformed signals are subsequently used as input to the estimator.

\section{The estimator}

The axial velocity $v_{z}$ estimates are based on the autocorrelation approach [36], and the two transverse velocity components are found by using the TO method [15] [16]. Since the applied array is geometrically identical in the transverse and lateral direction, the same apodization profile was applied for both cases, implying that $d_{x}=d_{y}$, which yields similar transverse wavelengths $\lambda_{x}=\lambda_{y}$. The estimator for calculating $v_{x}$ is therefore also valid for $v_{y}$, when beamforming in the orthogonal directions. The aliasing limit for this estimator is

$$
v_{x_{\max }}=\frac{\lambda_{x}}{4 k} f_{p r f}
$$

where $f_{p r f}$ is the pulse repetition frequency, $\lambda_{x}$ the transverse wavelength and $k$ the lag used in the autocorrelation.

The described TO method was used for velocity estimation in the simulation study and in the experimental setup.

\section{TRANSVERSE OSCILLATION ON A RC ARRAY}

Implementing $\mathrm{TO}$ on a $\mathrm{RC}$ array requires a few modifications compared to a fully addressed 2-D array, and this section explains how it was done.

\section{A. Transmission Sequences}

In this study, 3-D vector flow was estimated either in points along the axial $(0,0, z)$ direction (M-mode) or in points along several lines in the $z y$-plane (Steered). A total of 5 beamformed lines are needed to estimate 3-D vector flow along a line with TO. But since it is not possible to generate a focal point with a RC array, from which all 5 lines could be beamformed simultaneously the transmit sequences have to be interleaved. Two different flow transmit sequences were thus designed and used: 
1) M-mode: The M-mode sequence consisted of the two electronically focused emissions $R$ and $C$. The sequence was used, when 3-D vector flow estimates along one line was made. An illustration of the M-mode sequence is seen in Fig. 3. From the row emissions $R$, three of the five needed lines can be beamformed and the two velocity components $v_{x}$ and $v_{z}$ along the centerline can be derived. In the opposite scenario with transmit on the columns $C$ and receive on the rows, the $v_{y}$ and $v_{z}$ velocity components can be estimated. The applied emissions sequence is, thus, made by first transmitting on the rows and receiving on the columns and afterwards emitting with columns and receiving on the rows. The Mmode sequence was, thus, designed to alternately transmit the two emissions $R$ and $C$ in the following way.

$$
R \rightarrow C \rightarrow R \rightarrow C \rightarrow R \ldots
$$

This sequence is repeated $N_{e}$ times for each frame. By combining the two transverse estimates found from each transmit event with an axial estimate, the 3-D vector velocities are found.

2) Steered: The steered transmit sequence was used to estimate 3-D vector flow in the cross-sectional plane in a vessel. This sequence consisted of one focused emission $C_{1}$ using column elements and $N$ focused emissions $R_{i}$ using row elements, where $i=1 \ldots N$. 3-D vector flow was, thus, estimated in points along the $N$ steered directions in the $z y$-plane. The column emission generated a plane wave within the cross sectional $z y$-scan plane, whereas plane waves perpendicular to the scan plane were steered in the $z y$-plane when using the row elements, see Fig. 4. From the row transmit event $R_{i}$, the $v_{x_{i}}$ and $v_{z_{i}}$ velocity components could be estimated in points along the direction of the respective beamformed centerline. However, the $C_{1}$ column transmit event provided the required data for beamforming the lines needed for estimating all $v_{y_{i}}$ and $v_{z_{i}}$ velocity components, as this transmit event sonifies the $z y$-scan plane. The steered transmit sequence used is schematically written as

$$
\begin{aligned}
& C_{1} \rightarrow R_{1} \rightarrow R_{2} \rightarrow R_{3} \rightarrow \ldots R_{N} \\
& C_{1} \rightarrow R_{1} \rightarrow R_{2} \rightarrow R_{3} \rightarrow \ldots R_{N}
\end{aligned}
$$

Both sequences yields continuous data, which means that the distance between each identical emission type is equally distributed in time for all time. The advantages of continuous data are that very high frames rate can be obtained, and that dynamic ensemble lengths and echo canceling filters can be applied. The higher obtainable frame rate with continuous data occurs, since a sliding window can be applied on the beamformed data to generate one velocity estimate. The velocity estimate can be updated from each new similar emissions, since the new data can replace the oldest data in the estimator.

\section{B. Generating the transverse oscillation}

The TO method depends on generating two doubleoscillating fields, where one of the fields is a $90^{\circ}$ phase shifted version of the second one. If this is not the case, the velocity estimates will be biased [37]. In theory, the two fields should always be $90^{\circ}$ degree phase-shifted when $\lambda_{x}$ is calculated according to (1). However, (1) is only a mathematical approximation which leads to a deviation from the true $\lambda_{x}$ needed for a correct beamforming. The task is therefore to estimate $\lambda_{x}$ as precisely as possible to beamform the two $90^{\circ}$ phase-shifted double-oscillating fields.

This work uses the theoretical $\lambda_{x}$ to simulate two beamformed pulse-echo (PE) fields at the focal depth. A Hilbert transformation $\mathcal{H}\{\cdot\}$ of the left TO beamformed line $r_{\text {left }}$ was plotted along with the corresponding right TO line $r_{l e f t}$, see Fig. 5. If the two signals were $90^{\circ}$ phase shifted, the two lines would lay on top of each other. An optimization routine was run to find the $\lambda_{x}$ which minimized the lateral distance between the absolute values of the two peak amplitudes. This wavelength was considered the true $\lambda_{x}$ and was used in the beamformation.

A second transverse wavelength, $\bar{\lambda}_{x}$, was calculated from the 2-D spatio-temporal frequency spectrum [37]. $\bar{\lambda}_{x}$ was used as the transverse wavelength in the velocity estimator to reduce the bias [37]. The same transverse wavelengths were applied for beamforming and estimating $v_{y}$ due to transducer symmetry.

\section{Data Processing}

The raw RF data were processed on a Linux cluster. The same procedure was applied for all conducted measurements. Matched filtering was applied to the individual channel data by convolving it with the time-reversed emitted pulse to increase SNR. From each transmit event three lines were beamformed. Two of the lines, $r_{\text {left }}$ and $r_{\text {right }}$, were used to estimate the velocity component perpendicular to the tallest dimension of the receiving elements using the TO method, and the third line, $r_{\text {center }}$, was used to estimate the axial velocity with an autocorrelation approach [36]. Stationary echo canceling was performed by subtracting the mean value from the $N_{e}$ emissions in each frame for each beamformed line. After echo cancelling, the data were fed to the respective velocity estimator. By combining the estimated transverse velocity components, one from each transmit event, with one of the two independent axial estimates, a 3-D velocity vector along the direction of the respective beamformed centerline was obtained. An estimation plane was obtained by scan converting and interpolating the estimates.

\section{Simulation ENVIRONMENT}

The simulations in this study were performed with the ultrasound simulation program Field II [38] [39]. This section describes how the simulations were carried out, and which parameters were used.

Several parameters were used in the simulation environment. Some were kept fixed and others were varied in the 


\section{M-mode Sequence}

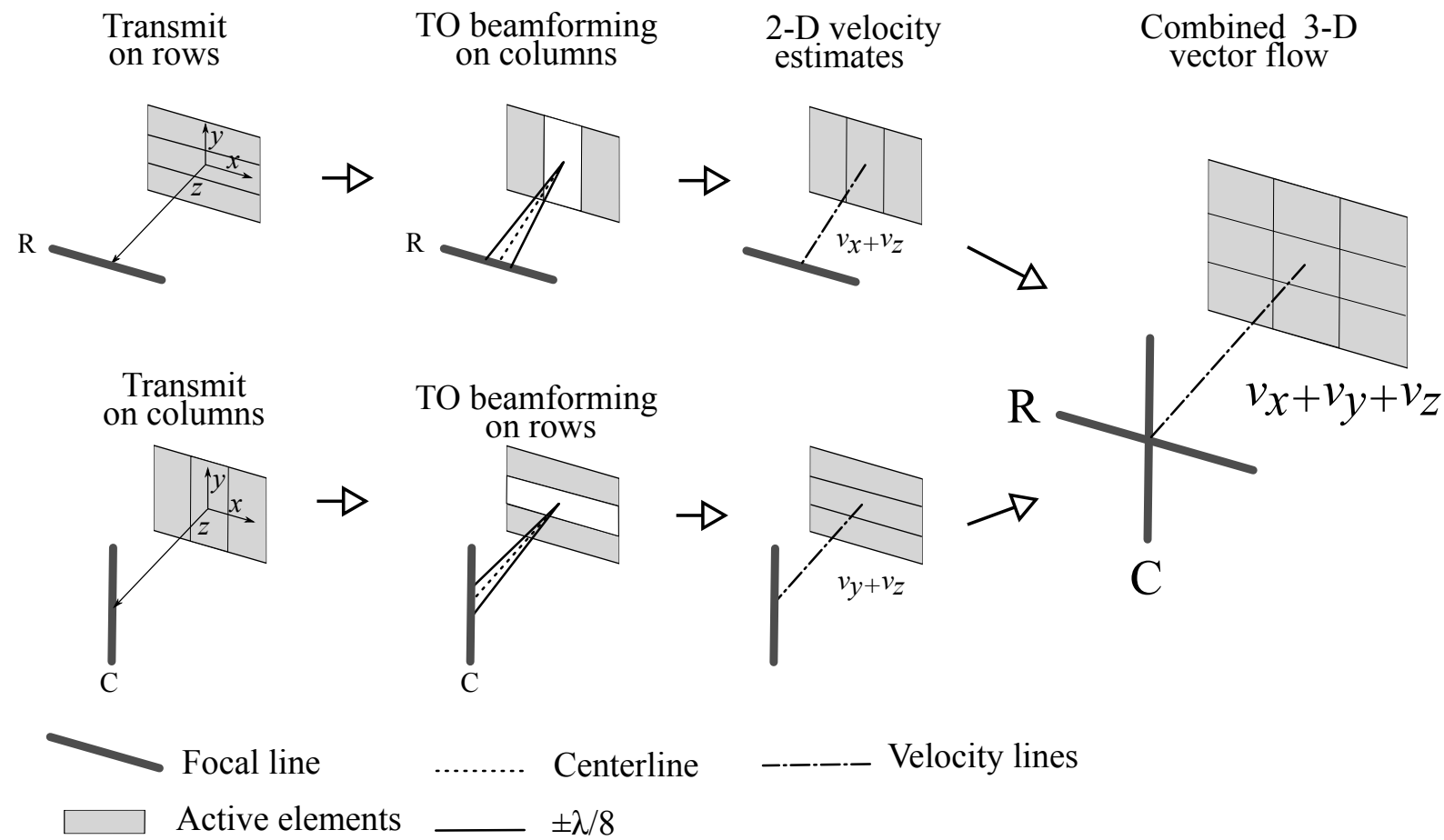

Figure 3: RC M-mode sequence for 3-D vector flow obtained along the center line with TO. The $R$ emission is made using row elements and generates a focal line in the $z x$-plane. Three lines are beamformed along the directions; $\left( \pm \lambda_{x}(z) / 8,0, z\right)$ and $(0,0, z)$ used for estimating $v_{x}$ and $v_{z}$. Subsequently a focal line in the orthogonal $z y$-plane is generated using the column elements $C$. By beamforming the lines $\left(0, \pm \lambda_{y}(z) / 8, z\right)$ and $(0,0, z), v_{y}$ and $v_{z}$ can be estimated. Finally, 3-D vector flow along the overlapping line is formed by combining the three estimated velocity components.

parameter study. The chosen parameters were varied to investigate the performance of the method. Parameters influencing the proposed method's angle independence, frame rate, intensity level, field-of-view, velocity range and accuracy were varied. The parameters with their default values (written in bold) are found in Table I.

A 2-D row-column array with integrated apodization was simulated with a center frequency, $f_{0}=3.0 \mathrm{MHz}$ similar to the one described in [29], [32]. Each dimension consisted of 62 elements with a pitch of $270 \mu \mathrm{m} \approx \lambda / 2$, where $\lambda$ is the wavelength in water with the given $f_{0}$. The kerf was set to $25 \mu \mathrm{m}$ or $\approx \lambda / 20$. At the end of each line element, a Hann roll-off element apodization of size $16 \times$ pitch was simulated.

A 6-cycles sinusoidal pulse was transmitted at $f_{0}$ from either all row or all column elements. As default, the emitted ultrasound was focused at $(x, y, z)=(0,0,30) \mathrm{mm}$. In the study where the steering angle $\theta_{z y}$ was varied in the $z y$-plane, the axial focus was always in $30 \mathrm{~mm}$ depth. The pulse repetition frequency $f_{p r f}$ was $3.0 \mathrm{kHz}$ as default.

A fixed symmetric Hann window spanning 62 elements was applied as apodization when transmitting the pulse from either all row or column elements. In receive, a symmetric Hann window apodization across all elements orthogonal to the transmitting aperture was applied for the axial velocity estimator. A similar procedure was followed when beamforming the two lines needed for estimating the transverse velocities, where only the apodization profile was changed to contain two symmetric Hann windowed apodization peaks of width $w=$ $25 \times$ pitch spaced by a distance $d$, of $35 \times$ pitch.

A $20 \times 20 \times 20 \mathrm{~mm}^{3}$ cubic phantom containing a cylindrical blood vessel $(\varnothing=12 \mathrm{~mm})$ located at $3 \mathrm{~cm}$ depth was defined for the simulations. Scatterers inside the cylinder were translated according to a circular symmetric parabolic velocity profile, and scatterers outside the cylinder were considered stationary. The magnitude of the scattering amplitude was $40 \mathrm{~dB}$ higher for the stationary scatters than for the moving blood cell mimicking scatterers. In total, 10000 scatterers were distributed in the phantom to ensure that more than 10 scatterer per resolution cell was present [40]. The peak velocity $v_{0}$ in the parabolic flow was $1 \mathrm{~m} / \mathrm{s}$ and propagation was in the $x$ direction as default. Rotation of the scatter phantom could be made around two axes; around the $y$-axis, denoted the beamto-flow angle $\alpha$ and rotation around the $z$-axis denoted $\beta$. In the default setup, no rotation was applied and a 90 degree beam-to-flow angle was present. An illustration of the similar experimental setup is seen in Fig. 6.

A total of 100 frames for each parameter configuration were simulated. With an ensemble length, $N_{e}$, of 32 , a total of $2 \times 32 \times 100$ emissions were used for each parameter setup. 100 velocity profiles were estimated in both the lateral and 


\section{Steered Sequence}

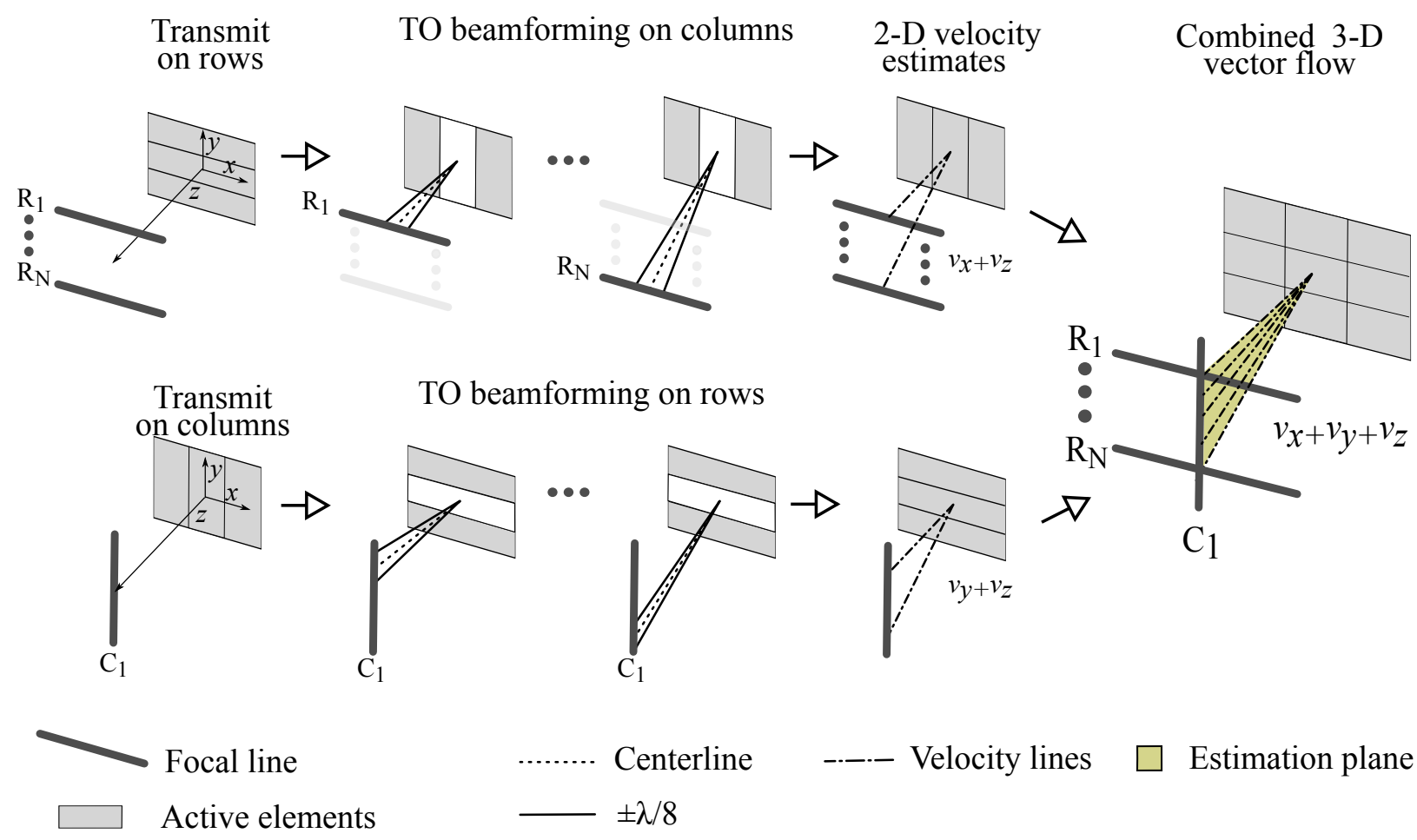

Figure 4: RC steered sequence for 3-D vector flow obtained in a cross sectional plane with TO. Compared to the M-mode sequence, the steered sequence differs in two ways; first, after each column emission $C_{1}$, multiple steered row emissions $R_{N}$ are emitted. From each row emission three lines are beamformed according to the steering directions and $v_{x}$ and $v_{z}$ can be estimated along each direction. Second, from a single column emission $C_{1}$, three lines are beamformed along each steering direction yielding $v_{y}$ and $v_{z}$ velocity estimates along the $N$ directions. 3-D vector flow is estimated in points along directions originating from the center of the aperture and through the intersection between the focal lines. The estimation plane is obtained when interpolating the combined 3-D vector flow estimates.

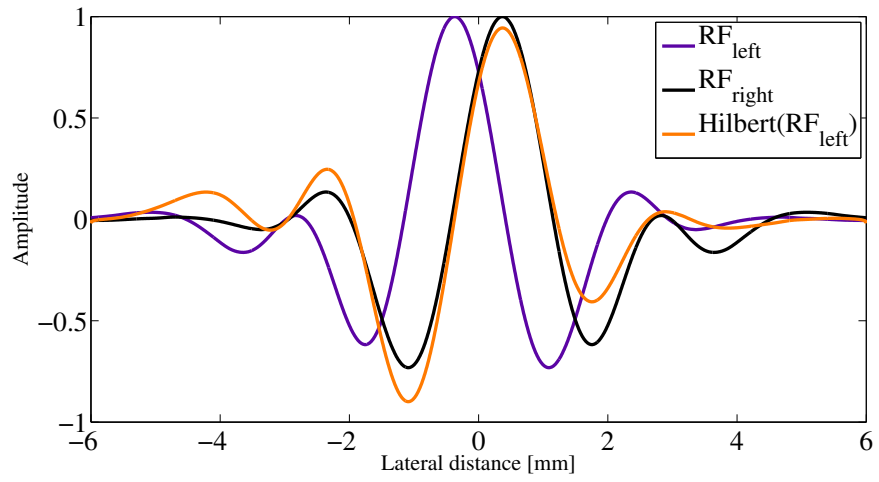

Figure 5: Beamformed $r_{\text {left }}$ and $r_{\text {right }}$ shown together with $\mathcal{H}\left\{r_{\text {left }}\right\}$ at $3 \mathrm{~cm}$ depth. A $90^{\circ}$ phase-shift is present, as $r_{\text {right }}$ and $\mathcal{H}\left\{r_{\text {left }}\right\}$ are on top of each other.

transverse direction and 200 velocity profiles in the axial directions due to the alternating between row and column emissions. All velocity profiles were independent as a random reposition of the scatterer was used after each frame.

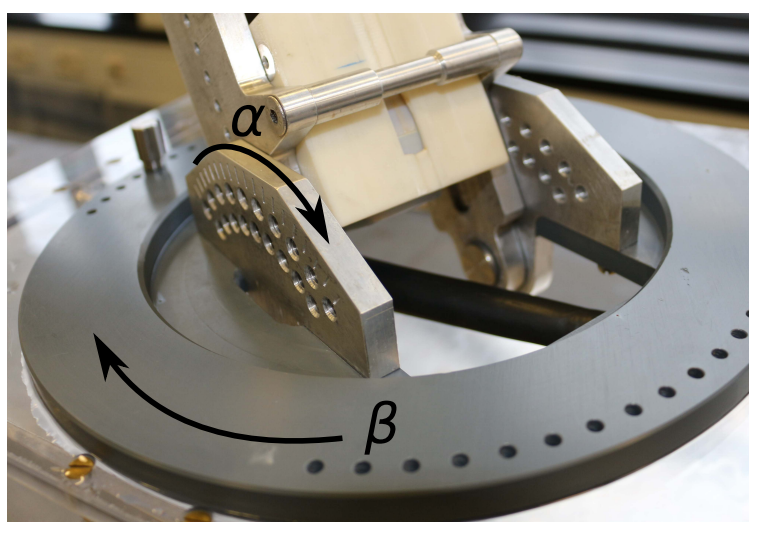

(a)

Figure 6: Illustration of the setup where a row-column transducer array is located $3 \mathrm{~cm}$ from the center of the vessel. In the default setup, the vessel is perpendicular to the transducer and the focal point is located in the center of the vessel. Two rotations of the 2-D prototype RC probe are applied; $\alpha$, which changes the beam-to-flow angle and $\beta$ which changes the flow rotation angle. 
Table I: Variables in Parameter Study

\begin{tabular}{llc}
\hline \hline Influencing & Parameter & Values \\
\hline \hline $\begin{array}{l}\text { Angle } \\
\text { independence }\end{array}$ & $\begin{array}{l}\text { Beam-to-flow angle } \alpha\left[^{\circ}\right] \\
\text { Flow angle } \beta\left[^{\circ}\right]\end{array}$ & $\begin{array}{c}\mathbf{9 0}, 75,60,45 \\
\mathbf{0}, 15,30,45\end{array}$ \\
\hline Frame rate & Ensemble length $N_{e}$ & $4,8,16, \mathbf{3 2}, 64$ \\
\hline Intensity & Pulse length [cycles] & $2,4,6,8$ \\
\hline Field-of-view & Steering angle $\theta_{z y}\left[^{\circ}\right]$ & $\mathbf{0 , 5}, \ldots, 40,45$ \\
\hline Velocity range & TO apo. width [pitch] & $1,5, \ldots, \mathbf{2 5}$ \\
& TO apo. peak spacing [pitch] & $25,27, \ldots, \mathbf{3 5}$ \\
\hline $\begin{array}{l}\text { Intensity } \\
\text { Accuracy } \\
\text { Accuracy }\end{array}$ & Transmit apo. & Heceive apo. (Axial) \\
Receive apo. (TO) & Hann, Tukey, Boxcar \\
\hline \hline
\end{tabular}

\section{EquipMEnT \& MATERIALS}

This section gives an introduction to all the equipment and materials used in the experimental setup. The 2-D RC prototype probe and the experimental ultrasound scanner SARUS [41] were used in all experiments in combination with one or more pieces of the described equipment.

\section{A. The 2-D RC prototype probe}

A $62+622-\mathrm{D}$ piezo RC prototype probe was used in the experiments with similar properties as the simulated array. Each row/column dimension consisted of 62 elements with the ends being dedicated for integrated apodization. Hence, the probe is denoted a $62+62 \mathrm{RC}$ probe. The probe had a center frequency $f_{0}$ of $3.0 \mathrm{MHz}$ which was also the transmit center frequency in the experimental setup. The pitch was 270 $\mu m$ and the kerf was $25 \mu m$ yielding an active aperture area of $1.67 \times 1.67 \mathrm{~cm}^{2}$. Four integrated edge apodization elements were used to reduce ghost echoes produced from the tall active line elements [32].

\section{B. SARUS}

All measurements were conducted with the experimental ultrasound scanner SARUS. Raw RF data from all 124 channels were stored with a sampling frequency of $35 \mathrm{MHz}$. The excitation voltage was $75 \mathrm{~V}$.

\section{Onda system}

The ONDA system consisted of a high precession AIMS III Scanning Tank (Onda, Sunnyvale, CA), a HGL-0400 (Onda, Sunnyvale, CA) hydrophone, an ATH-2000 attenuator, and a AH-2010 preamplifier. The equipment was assembled in the order with the hydrophone first, followed by the attenuator and the preamplifier, which was connected through a cable to SARUS. The assembled equipment was mounted in the scanning tank and was remotely controlled through a MATLAB interface and could move freely in all three spatial directions. The ONDA system was used to conduct the intensity measurements, as described in [42].

\section{Flow-rig}

An in-house built flow-rig system was used to validate the measured flow profile. The flow-rig contained a long inlet of $1.2 \mathrm{~m}$, which ensured that a steady laminar parabolic flow profile was present at the measuring site. Blood mimicking fluid was driven inside the system by a centrifugal pump in a closed loop circuit. At the measuring site, a straight rubber pipe $(\varnothing=12 \mathrm{~mm})$ was immersed into a water tank containing demineralized water. The volume flow $Q$, was measured with a MAG 1100 flow meter (Danfoss, Hasselager, Denmark). Peak velocity $v_{0}$ was estimated as $v_{0}=Q /\left(\pi R^{2}\right)$. The expected velocity profile could then be calculated as $v(r)=\left(1-\frac{r^{2}}{R^{2}}\right) v_{0}$, where $r$ is the distance from the center of the pipe. The transducer was placed in a fixture, where beam-to-flow angle $\alpha$, the flow rotation angle $\beta$ and the distance to the pipe were adjustable variables, see Fig. 6 . The fixture was then placed in the water tank and aligned to the center of the pipe. Several measurements with different combinations of $\alpha$ and $\beta$ where made.

\section{E. Pulsatile flow pump}

A second flow system (CompuFlow 1000, Shelley Medical Imaging Technologies, Toronto, Canada) was used to generate a pre-defined time-varying carotid flow waveform, where the pulse duration and flow rate of the waveform was adjustable. The manufacturer specified flow rate accuracy of the system was $\pm 3 \%$. The flow pump was connected to the straight-vessel phantom described in the following section.

\section{F. Straight-vessel phantom}

A customized tissue mimicking phantom (Dansk Fantom Service, Frederiksund, Denmark) containing a straight-vessel $(\varnothing=8 \mathrm{~mm})$ was used in combination with the flow pump. This phantom was only used when measuring pulsatile flow.

\section{Simulation PARAmeter StUdy}

To quantify and compare the performance when estimating the 3-D vector flow for different settings a statistical approach was used. All 100 estimated velocity profiles for a given setting were independent and comparable across parameter choice, as the same random number initialization seed was used for scatter distribution. At each sample point $z$ inside the vessel, the mean velocity $\bar{v}(z)$ and the standard deviation $\sigma(z)$ averaged over $N$ frames for each velocity component, was found as

$$
\begin{gathered}
\bar{v}(z)=\frac{1}{N} \sum_{i=1}^{N} v_{i}(z) \\
\sigma(z)=\sqrt{\frac{1}{N-1} \sum_{i=1}^{N}\left(v_{i}(z)-\bar{v}(z)\right)^{2} .}
\end{gathered}
$$

The mean relative bias $\tilde{B}$ between the estimated velocity and the expected velocity $v_{\mu}(z)$ at each depth was calculated as 


$$
\tilde{B}=\frac{1}{v_{0} N_{z}} \sum_{z=1}^{N_{z}}\left(\bar{v}(z)-v_{\mu}(z)\right),
$$

with $v_{0}$ beeing the theoretical peak velocity and where $N_{z}$ were all discrete samples located within $90 \%$ of the vertical vessel radius measured from its center. Boundary effects caused by e.g. echo canceling might give a wrong impression of the estimator's actual performance, which the $90 \%$ acceptance rule should help prevent. A similar relative mean standard deviation $\tilde{\sigma}$ was calculated as

$$
\tilde{\sigma}=\frac{1}{v_{0}} \sqrt{\frac{1}{N_{z}} \sum_{z=1}^{N_{z}} \sigma(z)^{2}} .
$$

The two quantitative performance metrics $\tilde{B}$ and $\tilde{\sigma}$ were used in the study for a comparison between different parameter settings.

\section{A. Pulse repetition frequency}

Although (2) states that the detectable velocity range scales with $f_{p r f}$, the applied values should be adjusted according to the expected velocities to reduce the standard deviation [21], [43]. In this simulation study, $f_{p r f}$ was scaled such that the expected peak velocity was $80 \%$ of the maximum detectable velocity. However, since three velocity components were estimated coherently from the same transmissions, individual maximum velocities could be detected, depending on the actual orientation of the vessel. As a consequence of this, $f_{p r f}$ was set to [3000 5000970013800$] \mathrm{Hz}$ for $\alpha=\left[\begin{array}{lll}90^{\circ} & 75^{\circ} 60^{\circ} 45^{\circ}\end{array}\right]$ respectively, to ensure that the aliasing limit was not exceeded for any of the three velocity components.

\section{B. Flow direction}

With the default parameter values listed in Table I, the flow phantom was rotated in different angle combinations. A total of 100 frames were recorded for each angle combination and data were processed as described in Section II. The ability to estimate angle-independent peak velocities from the 3-D velocity vector is shown in Fig. 7. For a $90^{\circ}$ beam-to-flow, the estimated peak velocity is found to be within $1.3-1.6 \%$ of $v_{0}$ for any investigated $\beta$ rotation. Decreasing $\alpha$ in general leads to a $\sigma$, which increases from $4.6 \%$ for $\alpha=90^{\circ}$, to $5.8 \%, 8.2 \%$ and $15.2 \%$ for $\alpha=75^{\circ}, 60^{\circ}$, and $45^{\circ}$ respectively. Results for the performance metrics are shown in Fig. 8a. For $\alpha=90^{\circ}$, $\tilde{B}_{v_{x}}$ stays between $0.2-3.5 \%$ with $\tilde{\sigma}_{v_{x}}$ between $4.6-5.7 \%$. As $\alpha$ increases, $\tilde{\sigma}_{v_{x}}$ and $\tilde{\sigma}_{v_{y}}$ increases to $9 \%$ for $\beta=15^{\circ}, 15 \%$ for $\beta=30^{\circ}$ and up to $20 \%$ when $\beta=45^{\circ}$ for both $\tilde{\sigma}_{v_{y}}$ and $\tilde{\sigma}_{v_{x}}$. The bias $\tilde{B}_{v_{y}}$ and $\tilde{B}_{v_{x}}$, however, only changes slightly from $\approx 0.1 \%$ and down to $-2.6 \%$ when $[\alpha ; \beta]=\left[45^{\circ} ; 45^{\circ}\right]$. A representative selection of velocity profiles from different rotation combinations are shown in Fig. 9. The figure shows how the standard deviation gradually increases for $v_{x}$ and $v_{y}$, which affects the final absolute velocity magnitude profile.

\section{Ensemble length}

The effect of the ensemble length $N_{e}$ was investigated in Fig. 8c. All estimates were derived from the same simulation, containing 100 frames each made from 2x64 emissions. The best estimates were found with the largest ensemble length $N_{e}=64$, where $\tilde{B}_{v_{x}}=1.8 \%$ and $\tilde{\sigma}_{v_{x}}=3.8 \%$ compared to $\tilde{B}_{v_{x}}=7.5 \%$ and $\tilde{\sigma}_{v_{x}}=8.1 \%$ for $N_{e}=8$. The same pattern was seen for $\tilde{B}_{v_{y}}$ and $\tilde{\sigma}_{v_{y}}$. A rapid increase in the standard deviation was seen when the ensemble length was lowered. This was expected, as the standard deviation is proportional to the included statistics and scales with $1 / \sqrt{N_{e}}$. However, a surprisingly high bias was found. A short ensemble length can increase the temporal resolution, but lowers the robustness of the estimator. This can especially be exploited when continuous data is present, such that the ensemble length is variable and adapts to the initial temporal variation of the flow.

\section{Pulse length}

The transmitted pulse length was varied from 2 to 8 cycles in steps of two cycles. $\tilde{B}_{v_{x}}$ increased from $-2.1 \%$ at two cycles and up to $2.6 \%$ when using 8 cycles. Standard deviation and bias for the remaining estimates were almost unaffected by changing the pulse length, presumably because no flow was present in these directions. Results are shown in Fig. 8d.

\section{E. Apodization}

Different transmit and receive apodization shapes were investigated, and the findings are presented in Fig. 8e. In the direction of the flow, the lowest bias was found when a Hann window was used in transmit and a boxcar apodization was used in receive, here $\tilde{B}_{v_{x}}=-0.1 \%$. The standard deviation increased slightly for this combination, but was relatively unaffected of the transmit and receive apodization, since $\tilde{\sigma}_{v_{x}}$ for all combinations of using a Hann window in transmit was between $4.6 \%$ and $5.5 \%$. However, $\tilde{\sigma}_{v_{y}}$ was highly affected by the transmit apodization and was $5.6 \%, 11.7 \%$, $7.5 \%$ when receive apodization was fixed to a boxcar, and transmit varied between a Hann, boxcar and a Tukey window, respectively.

\section{F. Steering angles}

Focused emissions were steered in the $z y$-plane spanning from $0^{\circ}$ to $45^{\circ}$ in steps of $5^{\circ}$. To make a fair comparison, the simulated phantom was shifted a certain distance in the $y$-direction to ensure that the focal line was always placed in the center of the vessel. Velocity profiles were derived from each steering angle and compared to the expected value. The resulting performance metrics are shown in Fig. 8f. The estimator deteriorates significantly for angles $>20^{\circ}$, where $\tilde{B}_{v_{x}}$ increases in a negative direction from a stable level around $1-2 \%$. The explanation to the breakdown is found in the geometry of the RC array and the position of the integrated apodization. The returning echo from a scatterer placed outside the physical aperture $|x|>8.4 \mathrm{~mm}$ or $|y|>8.4 \mathrm{~mm}$ will first hit the integrated apodization element, which results in a damped 


\section{Beam-to-flow angle $\alpha\left[^{\circ}\right]$}

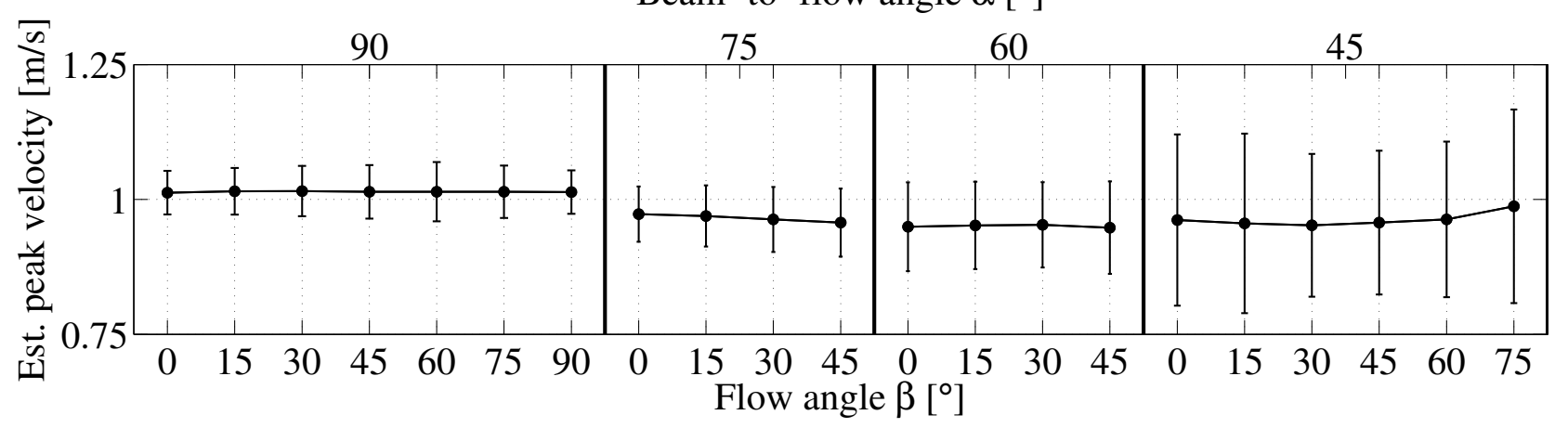

Figure 7: Peak velocities derived from the estimated 3-D vector velocities as a function of vessel orientation.

signal compared to a scatterer that was placed inside the physical aperture. The maximum dampening of the signal is found when the scatterer is placed outside the dimensions of the integrated apodization $|x|>12.7 \mathrm{~mm}$ or $|y|>12.7 \mathrm{~mm}$. For a $15^{\circ}$ steering angle, the center of the vessel was placed in $(8,0,30) \mathrm{mm}$, inside the physical aperture. For a $20^{\circ}$ steering angle the performance gradually breaks down, since the center of the vessel now is placed at $(11.9,0,30) \mathrm{mm}$, beneath the integrated roll-off apodization. This effect affects the performance more when increasing the steering angle, since a larger fraction of the scatterers are placed outside the physical aperture.

\section{G. TO spacing}

With a default TO peak width of 25 elements, the minimum element spacing between the two peaks was 25 and maximum 37 element before exceeding the aperture. The $\lambda_{x}$ used in the beamformation was found for each setup along with the optimized $\bar{\lambda}_{x}$ used in the velocity estimator as described in Section IV-B. A gradually improved performance was seen when increasing the separation between the two apodization peaks, see Fig. $8 \mathrm{~g}$. At a 25 element spacing, $\tilde{B}_{v_{x}}$ was $5.0 \%$ and improved to $1.3 \%$ for the 37 element spacing. Similarly, $\tilde{B}_{v_{y}}$ improved from $-0.6 \%$ to $-0.1 \%$. Likewise, $\tilde{\sigma}_{v_{x}}$ and $\tilde{\sigma}_{v_{y}}$ decreased from $6.8 \%$ and $8.4 \%$ to $4.8 \%$ and $5.9 \%$ respectively. Axial estimates were unaffected when varying TO specific parameters, as the estimator is decoupled from the TO method.

\section{H. TO width}

Varying the apodization width, while keeping the spacing between the peaks fixed, changes the transverse wavelength slightly. As the width decreases, $\lambda_{x}$ increases slightly. To make a fair comparison, both $\lambda_{x}$ and $\bar{\lambda}_{x}$ were estimated for each investigated width as described in Section IV-B. $\tilde{B}_{v_{y}}$ and $\tilde{\sigma}_{v_{y}}$ did not change significantly when varying the TO width, which probably should be attributed to the actual vessel orientation where $v_{y}=0 \mathrm{~m} / \mathrm{s}$, see Fig. $8 \mathrm{~h}$. $\tilde{B}_{v_{x}}$ changed from $2.4 \%$, when two delta functions were used in receive to $1.3 \%$ for a 25 elements wide Hann apodization. $\tilde{\sigma}_{v_{x}}$ changed similarly from $17.1 \%$ to $4.8 \%$.
Table II: Experimental parameter choice

\begin{tabular}{ll}
\hline \hline Parameter & Value \\
\hline Ensemble length $N_{e}$ & 32 \\
Pulse length & 8 \\
TO apo. width [pitch] & 25 \\
TO apo. peak spacing [pitch] & 35 \\
Transmit apo. & Hann \\
Receive apo. Axial/TO & Hann \\
\hline \hline
\end{tabular}

\section{Parameter study summary}

The effect on the velocity estimation performance was investigated when varying several parameters. Based on the presented findings, the following conclusions can be drawn: The best measuring position for the TO method on a 2-D $\mathrm{RC}$ array is found when a beam-to-flow angle of $90^{\circ}$ can be obtained, which is common in-vivo. The performance is decreasing as $\alpha$ is lowered, since the increase in $f_{p r f}$ (to avoid the aliasing limit) increases the standard deviation for an autocorrelation estimator [43]. A Hann apodization is preferred in transmit, whereas the shape of the receive apodization has less impact on the performance. The transmitted pulse length should be between 4 and 8 cycles and preferably to the lower end, when intensity levels are of concern. An optimal TO peak apodization should be as wide as possible and separated as much as possible. However, the actual shape needs to be optimized depending on the size of the applied array. Also, the expected velocity range needs to be taken into account when designing the apodization profiles. The number of emissions averaged over, $N_{e}$, should be 16 or above to ensure robust estimates. Accurate velocity estimates can be obtained for steered beams at angles $\leq 20^{\circ}$ where the absolute biases are $<$ $3 \%$ and $\tilde{\sigma}$ does not exceed $6 \%$. The estimator gradually breaks down when flow outside the physical aperture is examined.

Based on the simulation study, the suggested choice of parameters listed in Table II were fixed and used in the experimental validation described in the following sections.

\section{INTENSITY MEASUREMENTS}

Prior to the experimental validation, intensity measurements with the optimized parameter configuration were made to 
Beam-to-flow angle $\alpha\left[^{\circ}\right]$
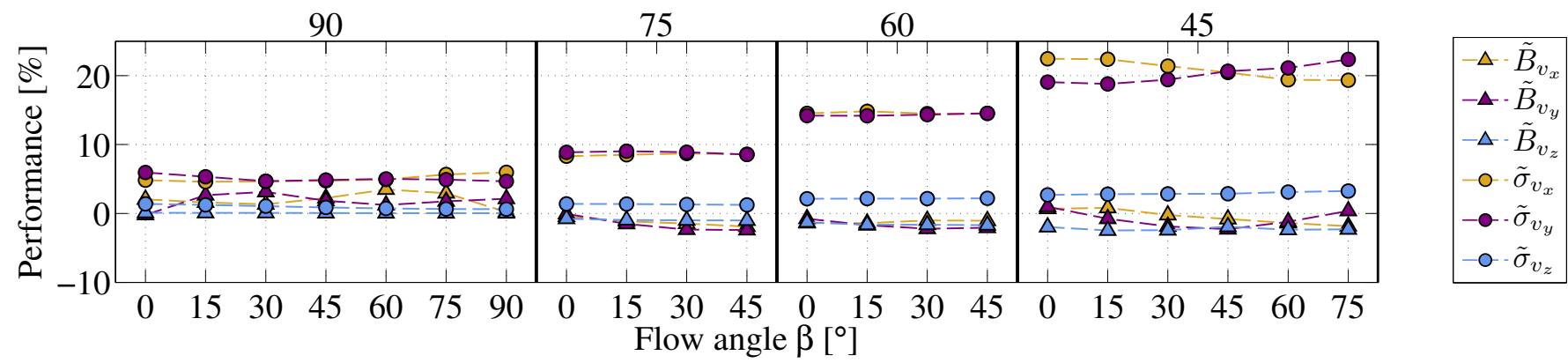

(a)

(b)

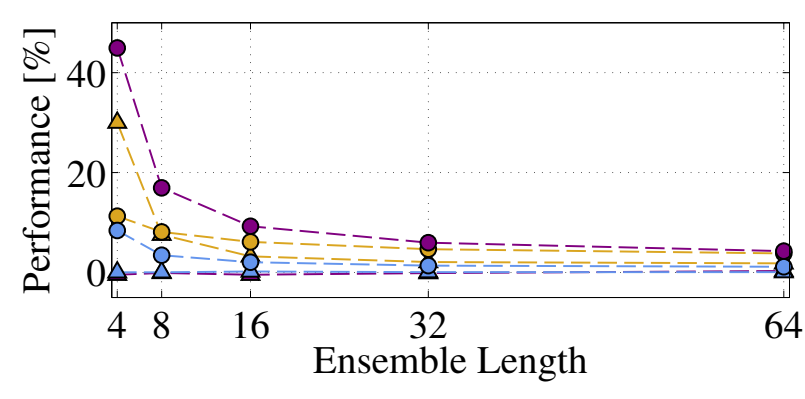

(c)

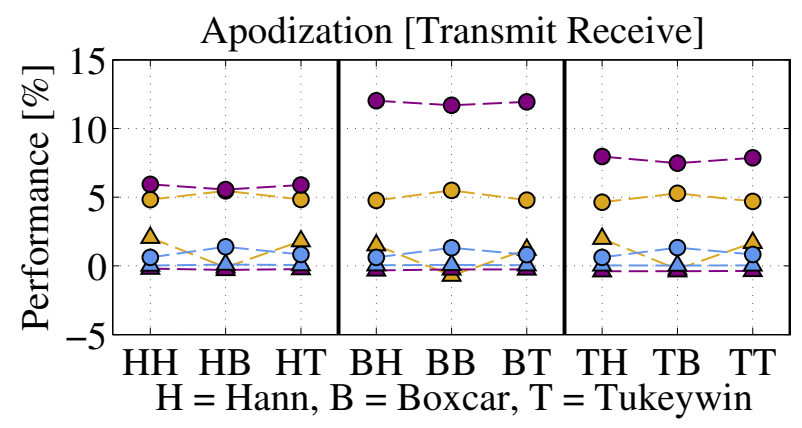

(e)

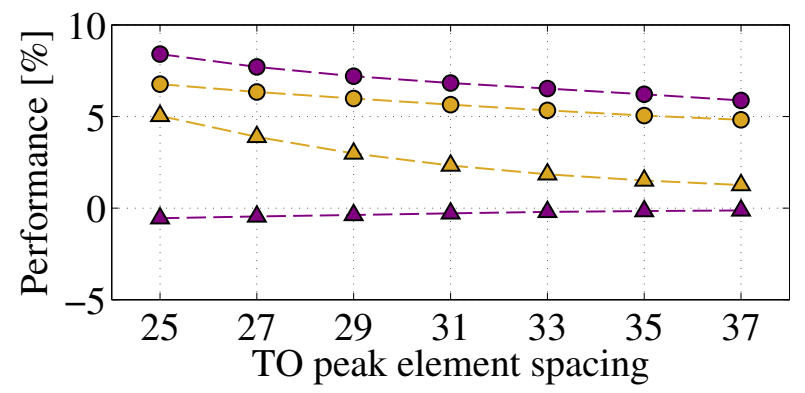

(g)

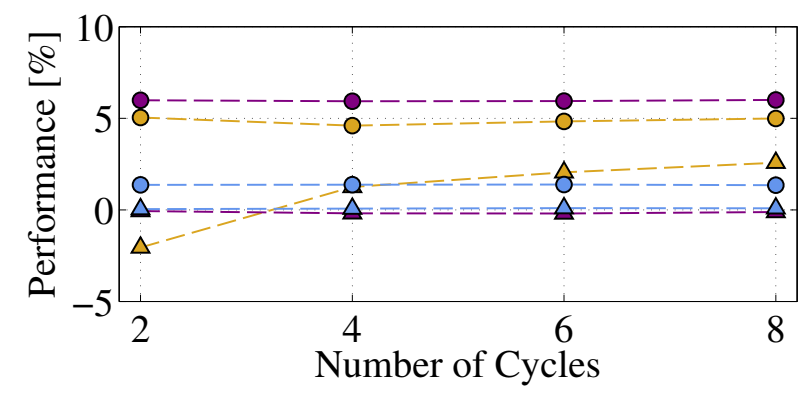

(d)

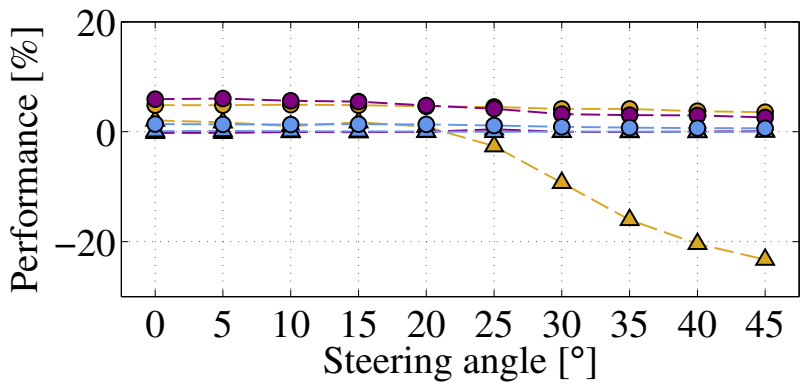

(f)

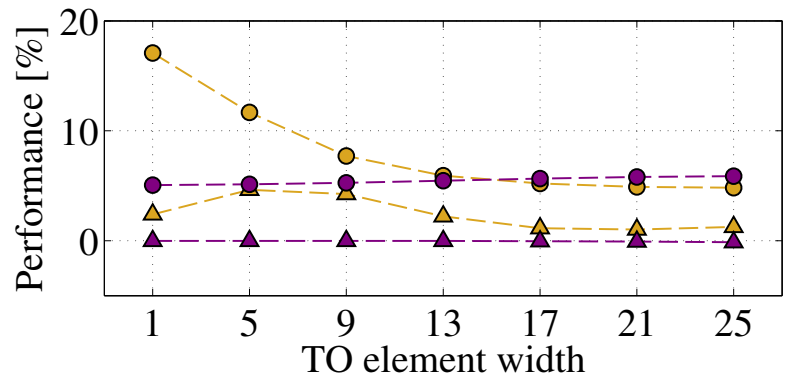

(h)

Figure 8: Results from the presented parameter study. An explanation to the legends is shown in b). Section VII gives a thorough review of the results.

confirm that the generated pressure field from the transmit sequence was as expected.

The transmit field for the M-mode sequence was measured in a plane at $z=30 \mathrm{~mm}$ from which the mechanical index (MI) and $I_{\text {spta.3 }}$ was calculated off line [42]. The estimated MI field is expected to be symmetric around the two focal lines. Furthermore, MI values along the focal lines, which are within the physical aperture, are supposed to be similar. The results shown in Fig. 10a confirms this, as a uniform MI along $(x, 0,30 \mathrm{~mm})$ and $(0, y, 30 \mathrm{~mm})$ of around 0.5 is found within the physical dimensions of the probe. Outside the focal lines, MI drops rapidly. The expected intensity field is also supposed 

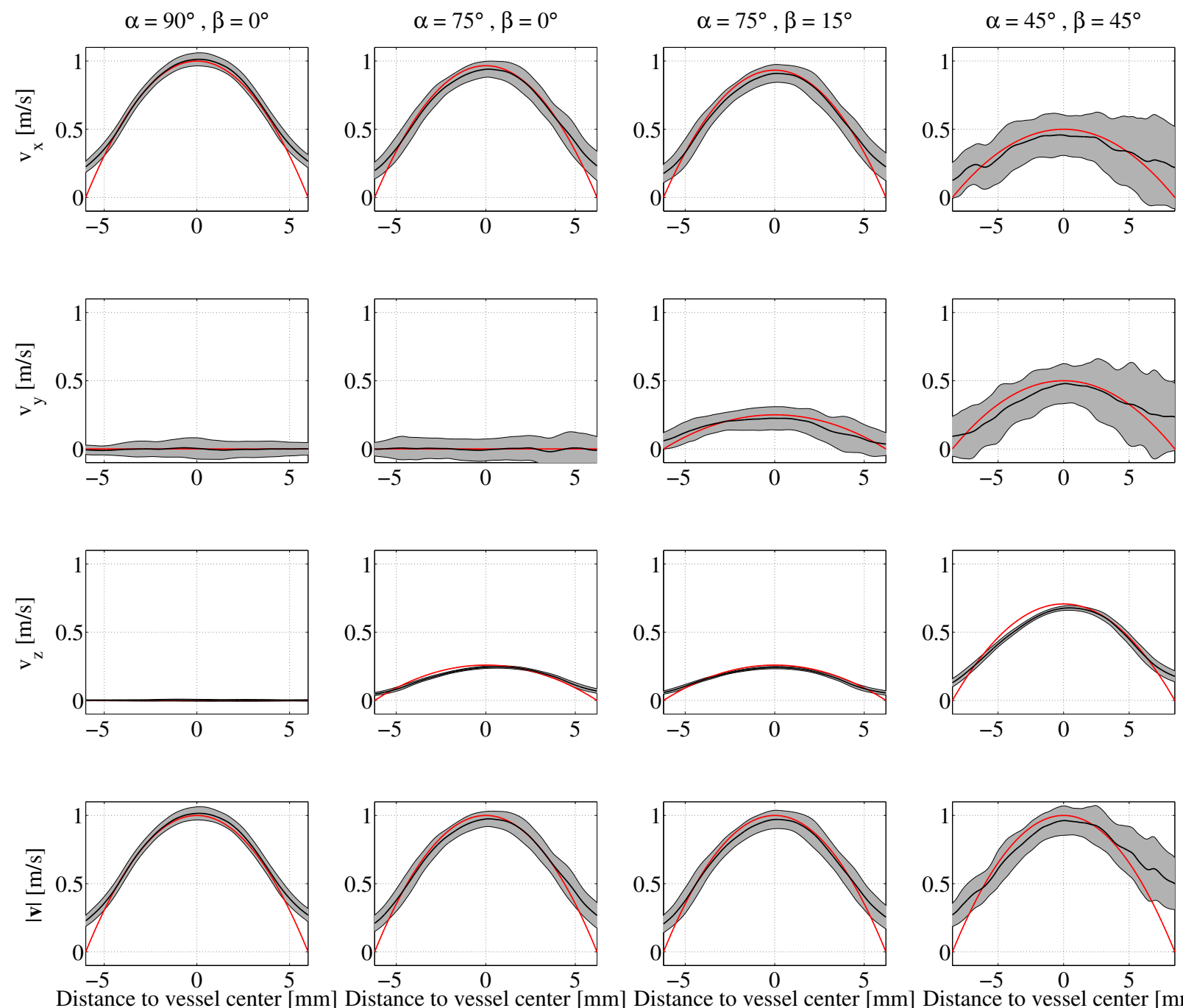

Figure 9: The decoupled estimated velocity component (black line) \pm one standard deviation (gray area) at a representative selection of different vessel rotation combinations are presented, along with the theoretical velocity component (red line). The bottom row shows the absolute velocity magnitude combined from the three velocity component estimates.

to be symmetric around both focal lines for $I_{\text {spta.3. }}$. However, since $I_{\text {spta. } 3}$ is composed by adding up the contribution from each emission, a peak in the intensity is expected in $(0,0$, $30 \mathrm{~mm}$ ) where the two focal lines are crossing. The peak intensity is supposed to be twice the value measured at the focal lines close to the end of the physical aperture, since both transmit events contribute equally to $I_{\text {spta.3 }}$ in $(0,0$, $30 \mathrm{~mm})$. The derated $I_{\text {spta.3 }}$ values are shown in Fig. 10b. A symmetric $I_{\text {spta.3 }}$ field is seen around the two focal lines with a peak centered in $(0,0,30 \mathrm{~mm})$. The peak $I_{\text {spta.3 }}$ is $14.0 \mathrm{~mW} / \mathrm{cm}^{2}$ and is reduced to $6.9 \mathrm{~mW} / \mathrm{cm}^{2}$ and $7.0 \mathrm{~mW} / \mathrm{cm}^{2}$ at $\pm 2 \mathrm{~mm}$ from the peak value in the elevation direction. Intensity measurements were performed at a $f_{p r f}=750 \mathrm{~Hz}$.

\section{ExPERIMENTAL Results}

This section describes the experimental measurements conducted. The presented measurements and results are presented in gradually increasing complexity.

\section{A. Flow-rig measurements}

1) M-mode: The M-mode flow sequence was used to estimate 3-D vector flow through the center line of the pipe in the flow-rig. Measurements from four representative combinations of $\alpha$ and $\beta$ were conducted. A total of $2 \times 32 \times 100$ emissions were used for each measurement setup. The peak velocity $v_{0}$ was set to $0.5 \mathrm{~m} / \mathrm{s}$ and the pulse repetition frequencies $f_{p r f}$ were $[1500,2500,6900]$ for the respective beam-to-flowangles $\alpha=\left[90^{\circ}, 75^{\circ}, 50^{\circ}\right.$, to ensure that the aliasing limit was not exceeded in the limiting velocity component $v_{z}$. The $f_{p r f}$ was halved compared to the simulations to maintain the same ratio between $v_{0}$ and $f_{p r f}$.

Results from the four measurements are shown in Fig. 11, where the individual velocity components are shown along with their respective standard deviation and the true profile. The estimated mean peak velocities calculated as $|\mathbf{v}|$ for each setup were $[48.5,50.4,50.6,58.2] \mathrm{cm} / \mathrm{s} \pm[3.0,5.9$, $3.5,8.7] \mathrm{cm} / \mathrm{s}$ for the respective flow combinations $[\alpha ; \beta]$ $=\left[90^{\circ} ; 0^{\circ}\right],\left[75^{\circ} ; 0^{\circ}\right],\left[75^{\circ} ; 15^{\circ}\right],\left[50^{\circ} ; 45^{\circ}\right] . \tilde{B}_{v_{x}}$ stays between 


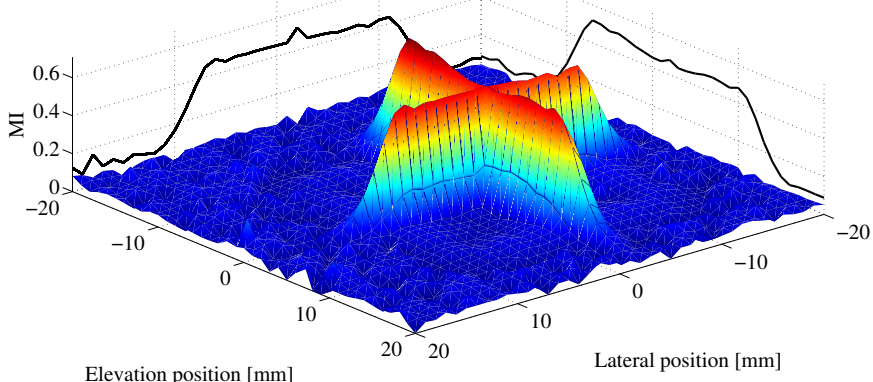

(a)

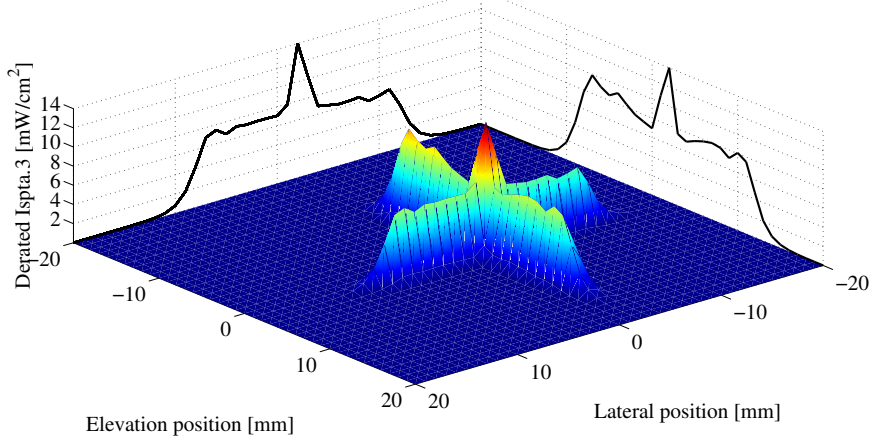

(b)

Figure 10: Intensity measurements of the M-mode flow sequence performed in the $y x$-plane at the depth of $30 \mathrm{~mm}$ at a $f_{p r f}=750 \mathrm{~Hz}$. a) Estimated MI. b) Estimated $I_{\text {spta.3 }}$.

$-3.1 \%$ and $-4.8 \%$ for the four constellations. Similarly $\tilde{B}_{v_{z}}$ stays between $-3.8 \%$ and $1.5 \%$ whereas $\tilde{B}_{v_{y}}$ increases from a level between $-0.9 \%$ and $2.1 \%$ in the first three setups to $-10.9 \%$ for $\left[50^{\circ} ; 45^{\circ}\right]$. The lowest mean relative standard deviation is found at $\left[90^{\circ} ; 0^{\circ}\right]$, where $\left(\tilde{\sigma}_{x}, \tilde{\sigma}_{y}, \tilde{\sigma}_{z}\right)=(8.7 \%$, $5.1 \%, 0.8 \%)$. The maximum standard deviation is found for the angle combination $\left[50^{\circ} ; 45^{\circ}\right]$, where $\left(\tilde{\sigma}_{x}, \tilde{\sigma}_{y}, \tilde{\sigma}_{z}\right)=$ $(19.9 \%, 21.3 \%, 6.5 \%)$. The large deviation in the estimated velocities compared to the expected, are supposed to be due to reverberation artifacts from the pipe.

2) Steered: With the same equipment and measurement setup, 3-D vector flow was measured in a cross sectional plane using the steered emission sequence. The sequence consisted of 11 steered row emissions spanning from $-12^{\circ}$ to $12^{\circ}$ in steps of $2.4^{\circ}$ and a single unsteered column emission. The $f_{\text {prf }}$ was $9.0 \mathrm{kHz}$ and a total of $32 \times 12 \times 100$ emissions were transmitted, corresponding to 100 frames when an ensemble length of 32 was used. A vector representation of the mean cross sectional 3-D vector flow averaged over all frames is seen in Fig. 12a. Based on the out-of-plane velocity component and the pipe area, the volumetric flow rate for each frame was estimated. The mean volumetric flow rate was $91.2 \mathrm{~L} / \mathrm{h} \pm$ $3.1 \mathrm{~L} / \mathrm{h}$ compared to the expected $102.6 \mathrm{~L} / \mathrm{h}$.

\section{B. Pulsating flow measurements}

1) M-mode: M-mode flow measurements were made using the flow pump and the straight-vessel phantom. A predefined carotid flow profile was chosen with a cycle time of $0.84 \mathrm{~s}$ and a flow rate of $2.54 \mathrm{~mL} /$ stroke $\pm 3 \%$. The transducer was placed $30 \mathrm{~mm}$ from the center of the vessel in a $90^{\circ}$ beamto-flow angle. At an $f_{p r f}$ of $750 \mathrm{~Hz}$ a total of $8.5 \mathrm{~s}$ were recorded by SARUS, corresponding to 10 full cycles. The sliding window was set to move in steps of 3 data points and an ensemble length of 32 was applied in the data processing. The predominant out-of-plane $v_{x}$ velocity component is shown as a function of time in Fig. 12b. Since the cycle time was fixed through the data acquisition, a time coherent addition of all 10 cycles was made. The mean velocity in the center of the vessel during each cycle was $11.3 \mathrm{~cm} / \mathrm{s} \pm 0.4 \mathrm{~cm} / \mathrm{s}$ and is seen in Fig. 12c.

2) Steered: With the same equipment and measurement setup, 3-D vector flow was measured in a cross sectional plane using the steered emission sequence. The sequence consisted of 11 steered row emissions spanning from $-8^{\circ}$ to $8^{\circ}$ in steps of $1.6^{\circ}$ and a single unsteered column emission. The $f_{p r f}$ was $9.0 \mathrm{kHz}$, which translates to 750 frames per second. A total of $4.3 \mathrm{~s}$ were recorded, corresponding to 5 cycles. The sliding window and ensemble length used in the processing was 3 and 32, respectively. The flow rate, based on the cross sectional vessel area and the out-of-plane velocity component averaged over 5 cycles was $2.3 \mathrm{~mL} /$ stroke $\pm 0.1 \mathrm{~mL} /$ stroke, compared to the expected $2.54 \mathrm{~mL} /$ stroke. The high frame rate captures the repeating pulsating behavior and the high velocities during peak-systole were $25.6 \mathrm{~cm} / \mathrm{s} \pm 0.9 \mathrm{~cm} / \mathrm{s}$ and the lower velocities during end-diastole were $1.1 \mathrm{~cm} / \mathrm{s} \pm$ $0.7 \mathrm{~cm} / \mathrm{s}$. A 3-D vector representation of the flow both during the peak-systole and end-diastole is seen in Fig. 13

\section{Discussion \& CONCLUSION}

The feasibility of implementing 3-D vector flow using the TO method on a prototype $62+62 \mathrm{RC}$ probe was demonstrated through various simulation setups and experimental measurements.

The experimental results were in good agreement with similar simulation setups. For instance, the estimated peak velocity had a mean relative bias of $1.3 \%$ and a relative mean standard deviation of $4 \%$ in the simulations and similar a mean relative bias of $-3.8 \%$ and a relative mean standard deviation of $5.9 \%$ in the measurements for the flow angle constellation $[\alpha ; \beta]=\left[90^{\circ} ; 0^{\circ}\right]$.

A cross sectional view of the 3-D vector flow in the flow-rig was estimated with a steered emissions sequence. Based on the cross sectional area and the out-of-plane velocity component, the flow rate was found to be $91.2 \mathrm{~L} / \mathrm{h} \pm 3.1 \mathrm{~L} / \mathrm{h}$ compared to the expected $102.6 \mathrm{~L} / \mathrm{h}$.

Evaluation of the performance, when estimating velocities in time-varying complex flow, was made with the flow pump. The results showed the pulsating flow pattern, and the estimated mean velocity in the center of the vessel during one period was $11.3 \mathrm{~cm} / \mathrm{s} \pm 0.4 \mathrm{~cm} / \mathrm{s}$, when averaging over 10 periods. Furthermore, both the slow and fast flow could be estimated in a cross-sectional scan plane and the mean flow rate from 5 cycles was found to be $2.3 \mathrm{~mL} / \mathrm{stroke} \pm$ $0.1 \mathrm{~mL} / \mathrm{stroke}$ with a negative bias of $9.7 \%$. Both pulsating 

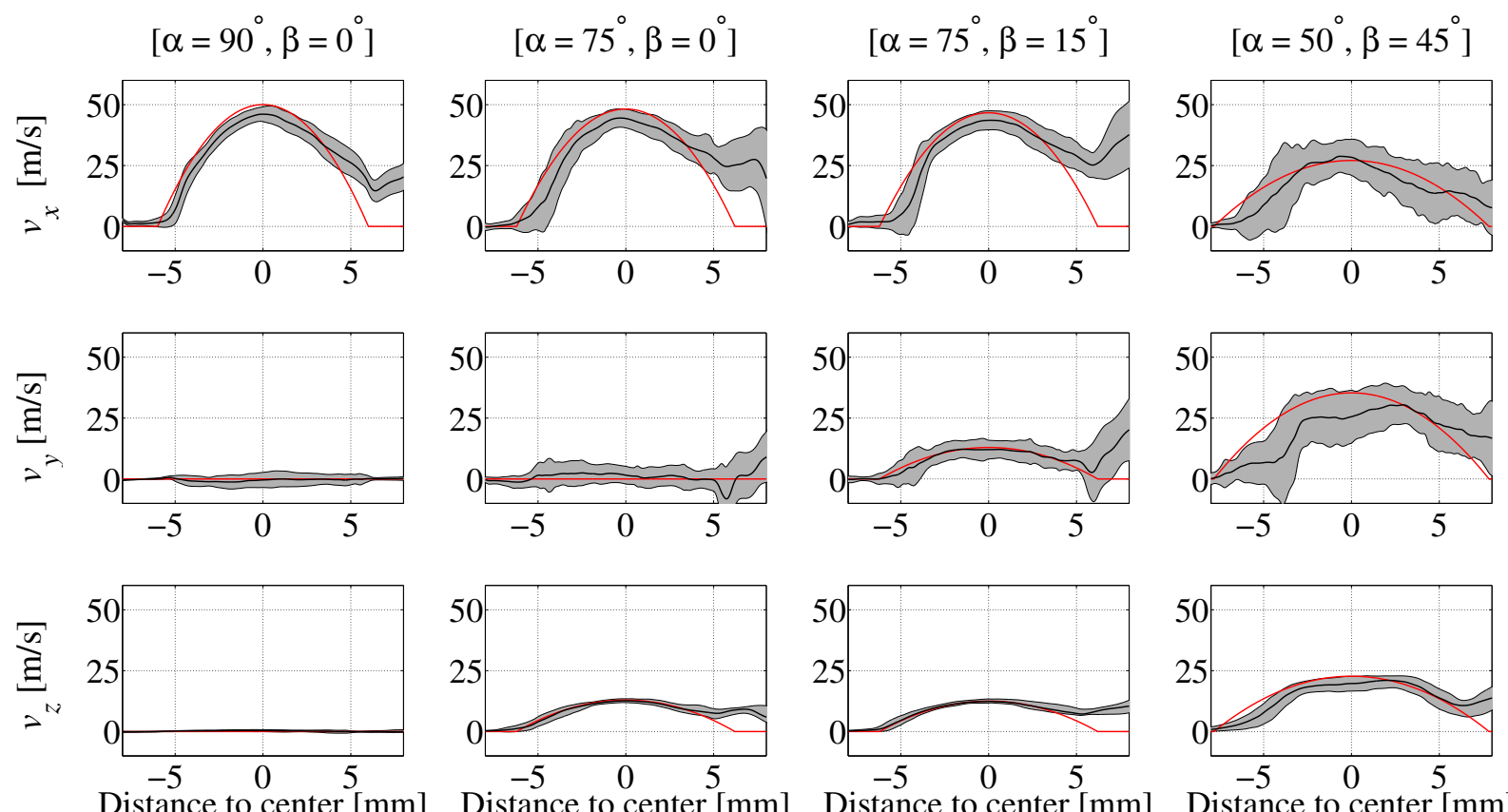

Distance to center $[\mathrm{mm}]$

Distance to center $[\mathrm{mm}]$

Distance to center [mm]

Figure 11: Individual mean estimated velocity components (black line) \pm one standard deviation (gray area) at a representative selection of different flow rotation combinations are presented with the expected profiles (red line).

flow results had a standard deviation below 5\%, which shows that the estimates are consistent and reproducible. In general a negative bias was found both in the simulations and in the experimental exploration. Further investigation of this behavior is needed to determine whether it is related to the estimation of the transverse wavelength or influenced by other effects.

The relatively low experimental peak velocities $(\sim 25-30$ $\mathrm{cm} / \mathrm{s}$ ) compared to the peak-systole in the common carotid artery $(\sim 1 \mathrm{~m} / \mathrm{s})$ were chosen to avoid the production and circulation of air bubbles in the system, which might corrupt the estimates. The presented method is not limited to slow flow, hence realistic peak velocities in vivo of $1 \mathrm{~m} / \mathrm{s}$ can be obtained by adjusting $f_{p r f}$, the number of steering angles, and the transverse wavelengths to match the flow conditions.

Intensity measurements with the RC probe showed that focused emissions generated plane waves, which sonified lines spanning the tall dimension of an element. The results of the intensity measurements forms the basis of the attainable fieldof-view for vector flow imaging using the applied prototype $\mathrm{RC}$ probe. With the current probe design, vector flow can be obtained in an an area limited to a box of approximately 20 $\mathrm{mm} \times 20 \mathrm{~mm}$ and the $z$-axis.

The presented results demonstrate that 3-D vector flow can be obtained with only 124 active elements, if they are positioned in a row-column wise scenario. Since most scanners on the marked can sample at least 128 channels simultaneously, real time 3-D vector flow estimation no longer requires a customized research ultrasound scanner and a fully addressed matrix probe, but only a commercial scanner with access to raw channel data and a RC probe.

For in vivo applications, when e.g. measuring in the common carotid artery, several adjustments can be made to the presented steered sequence to cope with velocities on the order of $1 \mathrm{~m} / \mathrm{s}$. For instance, the TO peak element spacing can be shortened (as described in Section VII-G) to yield a larger lateral wavelength which translates to a higher detectable $v_{\max }$ according to 2 . Furthermore, since the proposed method uses a phase estimator which ranges from $[-\pi: \pi]$ which translates to a velocity range of $\left[-v_{\max }: v_{\max }\right]$, a phase shift of e.g. $\frac{\pi}{2}$ can be applied such that the phase range changes to $\left[-\frac{\pi}{2}\right.$ $\left.: \frac{3}{2} \pi\right]$, which shifts the detectable velocity range to $\left[-\frac{1}{2} v_{\max }\right.$ $\left.: \frac{3}{2} v_{\max }\right]$ for better estimation of larger positive velocities. A feasible sequence for estimating realistic in vivo velocities could contain $N=11$ steered row emissions and one column emission, have a TO peak spacing of 31 elements and be emitted at a $f_{p r f}=15 \mathrm{kHz}$. At $3 \mathrm{~cm}$ the detectable positive velocity $v_{\max }$ would then be

$$
v_{x_{\text {max }}}=\frac{\lambda_{x}}{4 k} \frac{f_{p r f}}{N+1}=\frac{0.40 \mathrm{~cm}}{4 * 1} \frac{15 \mathrm{kHz}}{11+1}=125 \mathrm{~cm} / \mathrm{s},
$$

which could be increased to $188 \mathrm{~cm} / \mathrm{s}$ by applying a $\frac{\pi}{2}$ phase shift. The presented sequence would produce ultra fast vector flow estimates of 1250 frames per second.

Future work is planned to validate the clinical application of 3-D vector flow on the RC probe, when a permission by the science ethics board has been granted for in vivo usage.

\section{ACKNOWLEDGMENT}

This work was supported by grant 82-2012-4 from the Danish National Advanced Technology Foundation and by BK Ultrasound Aps. The authors would also like to thank BK Ultrasound Aps. and Sound Technology Inc., for development of the RC probe.

\section{REFERENCES}

[1] G. M. von Reutern, M. W. Goertler, N. M. Bornstein, M. D. Sette, D. H. Evans, A. Hetzel, M. Kaps, F. Perren, and et al., "Grading carotid stenosis using ultrasonic methods," Stroke, vol. 43, no. 3, pp. 916-921, 2012 . 


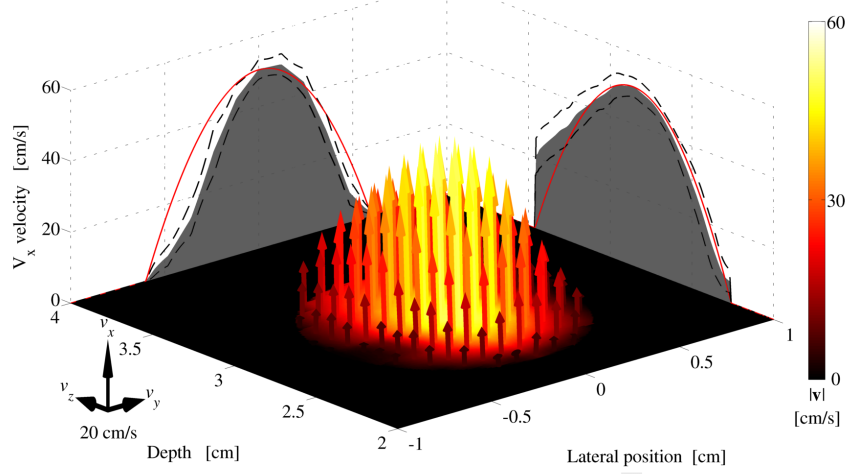

(a)

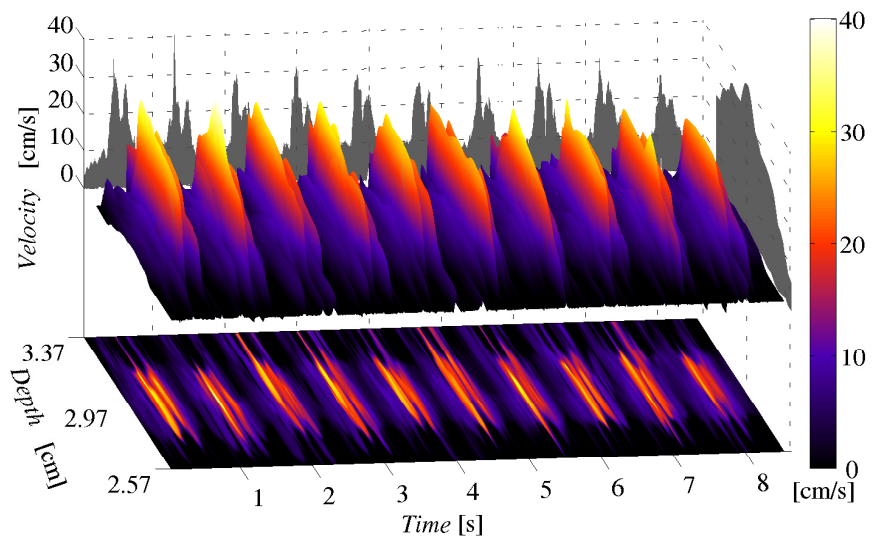

(b)

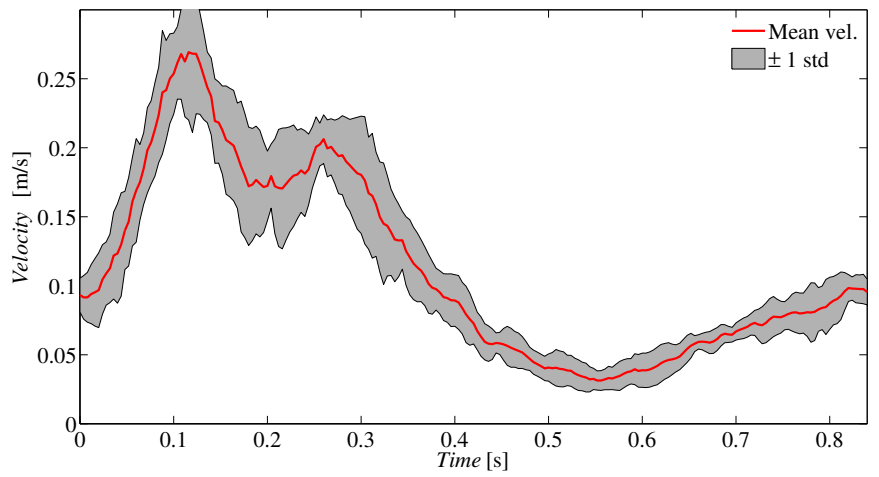

(c)

Figure 12: Cross sectional mean 3-D vector flow averaged over 100 frames. The magnitude and direction of the flow (at a certain position) is depicted by the length and the color of the arrows. The shaded gray areas represents the projection of the flow in the respective direction with its standard deviation (dotted line). The theoretical flow profiles are illustrated by the red lines. b) M-mode of the out-of-plane $v_{x}$ velocity component measured in a pulsating carotid flow waveform. c) Mean $v_{x}$ velocity in the center of the vessel throughout a period averaged over 10 cycles.

[2] K. L. Gould, "Quantification of coronary artery stenosis in vivo," Circ Res., vol. 57, no. 3, pp. 341-353, 1985.

[3] P. A. Picot and P. M. Embree, "Quantitative volume flow estimation using velocity profiles," IEEE Trans. Ultrason., Ferroelec., Freq. Contr., vol. 41, pp. 340-345, 1994.

[4] E. G. Grant, C. B. Benson, G. L. Moneta, A. V. Alexandrov, J. D. Baker, E. I. Bluth, B. A. Carroll, and et al., "Carotid artery stenosis: gray-scale and doppler us diagnosis - society of radiologists in ultrasound consensus conference," Radiology, vol. 229, no. 2, pp. 340-346, 2003, m. Eliasziw and J. Gocke and B. S. Hertzberg.

[5] R. W. Gill, "Measurement of blood flow by ultrasound: Accuracy and sources of error," Ultrasound Med. Biol., vol. 11, pp. 625-641, 1985.

[6] J. Jensen, J. B. Olesen, M. B. Stuart, P. M. Hansen, M. B. Nielsen, and J. A. Jensen, "Vector velocity volume flow estimation: Sources of error and corrections applied for arteriovenous fistulas," Ultrasonics, vol. 70, pp. 136-146, 2016.

[7] G. E. Trahey, J. W. Allison, and O. T. von Ramm, "Angle independent ultrasonic detection of blood flow," IEEE Trans. Biomed. Eng., vol. BME-34, pp. 965-967, 1987.

[8] S. Fadnes, I. K. Ekroll, S. A. Nyrnes, H. Torp, and L. Løvstakken, "Robust angle-independent blood velocity estimation based on dualangle plane wave imaging," IEEE Trans. Ultrason., Ferroelec., Freq. Contr., vol. 62, no. 10, pp. 1757-1767, October 2015.

[9] J. A. Jensen, "Directional velocity estimation using focusing along the flow direction: I: Theory and simulation," IEEE Trans. Ultrason., Ferroelec., Freq. Contr., vol. 50, pp. 857-872, 2003.

[10] J. A. Jensen and R. Bjerngaard, "Directional velocity estimation using focusing along the flow direction: II: Experimental investigation," IEEE Trans. Ultrason., Ferroelec., Freq. Contr., vol. 50, pp. 873-880, 2003.

[11] B. Dunmire, K. W. Beach, K.-H. Labs., M. Plett, and D. E. Strandness, "Cross-beam vector Doppler ultrasound for angle independent velocity measurements," Ultrasound Med. Biol., vol. 26, pp. 1213-1235, 2000.

[12] M. Tanter, J. Bercoff, L. Sandrin, and M. Fink, "Ultrafast compound imaging for 2-D motion vector estimation: application to transient elastography," IEEE Trans. Ultrason., Ferroelec., Freq. Contr., vol. 49, pp. 1363-1374, 2002.

[13] D. Garcia, J. C. del Alamo, D. Tanne, R. Yotti, C. Cortina, E. Bertrand, J. C. Antoranz, E. Perez-David, R. Rieu, F. Fernandez-Aviles, and J. Bermejo, "Two-dimensional intraventricular flow mapping by digital processing conventional color-doppler echocardiography images," IEEE Trans. Med. Imag., vol. 29, no. 10, pp. 1701-1713, 2010.

[14] S. Ricci, L. Bassi, and P. Tortoli, "Real-time vector velocity assessment through multigate Doppler and plane waves," IEEE Trans. Ultrason., Ferroelec., Freq. Contr., vol. 61, no. 2, pp. 314-324, 2014.

[15] J. A. Jensen and P. Munk, "A new method for estimation of velocity vectors," IEEE Trans. Ultrason., Ferroelec., Freq. Contr., vol. 45, pp. 837-851, 1998.

[16] J. A. Jensen, "A new estimator for vector velocity estimation," IEEE Trans. Ultrason., Ferroelec., Freq. Contr., vol. 48, no. 4, pp. 886-894, 2001.

[17] M. Lenge, A. Ramalli, P. Tortoli, C. Cachard, and H. Liebgott, "Planewave transverse oscillation for high-frame-rate 2-D vector flow imaging," IEEE Trans. Ultrason., Ferroelec., Freq. Contr., vol. 62, no. 12, pp. 2126-2137, December 2015.

[18] I. A. Hein, "3-D flow velocity vector estimation with a triple-beam lens transducer - experimental results," IEEE Trans. Ultrason., Ferroelec., Freq. Contr., vol. 44, pp. 85-95, 1997.

[19] S. Berg, H. Torp, B. O. Haugen, and S. Samstad, "Volumetric blood flow measurement with the use of dynamic 3-dimensional ultrasound color flow imaging," J. Am. Soc. Echocardiog., vol. 13, no. 5, pp. 393-402, 2000.

[20] M. J. Pihl and J. A. Jensen, "A transverse oscillation approach for estimation of three-dimensional velocity vectors. Part I: Concept and simulation study," IEEE Trans. Ultrason., Ferroelec., Freq. Contr., vol. 61, pp. 1599-1607, 2014.

[21] M. J. Pihl, M. B. Stuart, B. G. Tomov, M. F. Rasmussen, and J. A. Jensen, "A transverse oscillation approach for estimation of threedimensional velocity vectors. Part II: Experimental validation,” IEEE Trans. Ultrason., Ferroelec., Freq. Contr, vol. 51, no. 10, pp. 1608$1618,2014$.

[22] J. Provost, C. Papadacci, J. E. Arango, M. Imbault, M. Fink, J. L. Gennisson, M. Tanter, and M. Pernot, "3-D ultrafast ultrasound imaging in vivo," Phys. Med. Biol., vol. 59, no. 19, pp. L1-L13, 2014.

[23] C. E. Morton and G. R. Lockwood, "Theoretical assessment of a crossed electrode 2-D array for 3-D imaging," in Proc. IEEE Ultrason. Symp., 2003, pp. 968-971.

[24] C. E. M. Démoré, A. Joyce, K. Wall, and G. Lockwood, "Real-time volume imaging using a crossed electrode array," IEEE Trans. Ultrason., Ferroelec., Freq. Contr., vol. 56, no. 6, pp. 1252-1261, 2009.

[25] C. H. Seo and J. T. Yen, "64 x 64 2-D array transducer with row-column addressing," in Proc. IEEE Ultrason. Symp., vol. 1, 2006, pp. 74-77. 

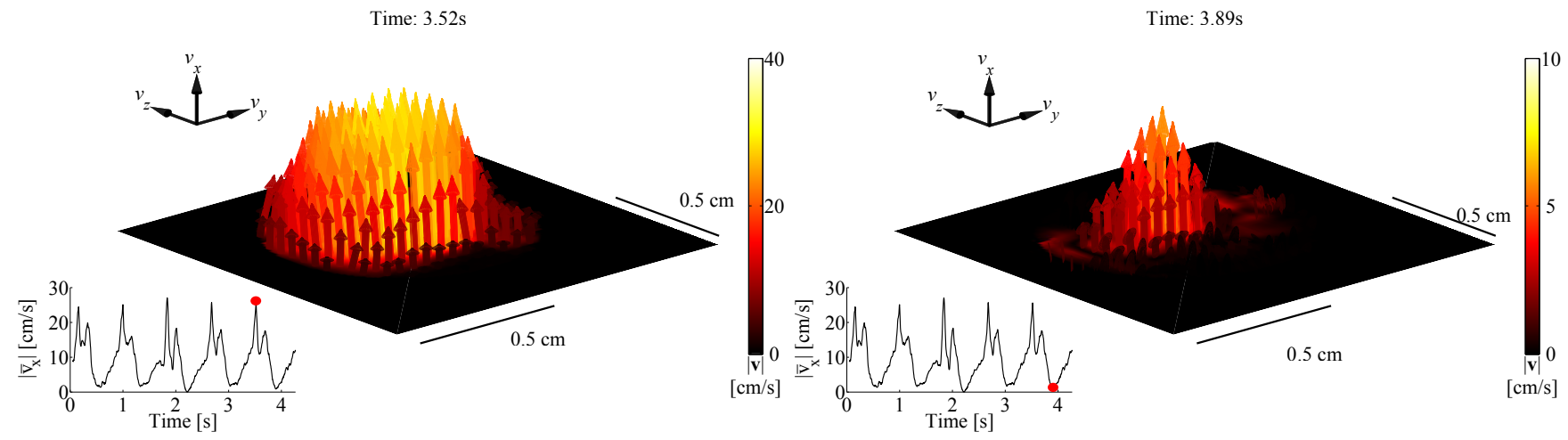

(a)

(b)

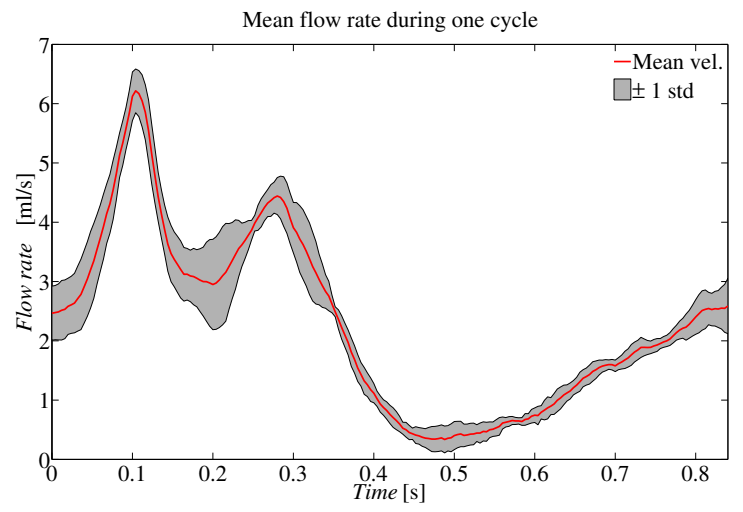

(c)

Figure 13: Cross sectional 3-D vector flow representation estimated in the pulsating setup during (a) peak-systole and (b) end-diastole. Please note that the velocity scale during peak-systole is four times larger than for the end-diastole. c) mean flow rate with std averaged across 5 cycles. A video of the complete flow is show in video 1 .

[26] J. T. Yen, C. H. Seo, S. I. Awad, and J. S. Jeong, "A dual-layer transducer array for 3-D rectilinear imaging," IEEE Trans. Ultrason., Ferroelec., Freq. Contr., vol. 56, no. 1, pp. 204-212, 2009.

[27] A. I. H. Chen, L. L. P. Wong, A. S. Logan, and J. T. W. Yeow, "A CMUTbased real-time volumetric ultrasound imaging system with row-column addressing," in Proc. IEEE Ultrason. Symp., oct 2011, pp. 1755-1758.

[28] A. Sampaleanu, P. Zhang, A. Kshirsagar, W. Moussa, and R. Zemp, "Top-orthogonal-to-bottom-electrode (TOBE) CMUT arrays for 3-D ultrasound imaging." IEEE Trans. Ultrason., Ferroelec., Freq. Contr., vol. 61, no. 2, pp. 266-276, 2014.

[29] T. L. Christiansen, M. F. Rasmussen, J. P. Bagge, L. N. Moesner, J. A. Jensen, and E. V. Thomsen, "3-D imaging using row-column-addressed arrays with integrated apodization - part II: Transducer fabrication and experimental results," IEEE Trans. Ultrason., Ferroelec., Freq. Contr., vol. 62, no. 5, pp. 959-971, 2015.

[30] S. Holbek, T. L. Christiansen, M. F. Rasmussen, M. B. Stuart, E. V. Thomsen, and J. A. Jensen, "3-D vector velocity estimation with rowcolumn addressed arrays," in Proc. IEEE Ultrason. Symp., 2015, pp. $1-4$.

[31] M. F. Rasmussen and J. A. Jensen, "3D ultrasound imaging performance of a row-column addressed 2D array transducer: a simulation study," in Proc. SPIE Med. Imag., 2013, pp. 1-11, 86750C.

[32] M. F. Rasmussen, T. L. Christiansen, E. V. Thomsen, and J. A Jensen, "3-D imaging using row-column-addressed arrays with integrated apodization - Part I: Apodization design and line element beamforming," IEEE Trans. Ultrason., Ferroelec., Freq. Contr., vol. 62, no. 5, pp. 947-958, 2015.

[33] H. Liebgott, A. Basarab, P. Gueth, D. Friboulet, and P. Delachartre, "Transverse oscillations for tissue motion estimation," Ultrasonics, vol. 50, no. 6, pp. 548-555, 2010.

[34] H. Liebgott, J. Fromageau, J. E. Wilhjelm, D. Vray, and P. Delachartre, "Beamforming scheme for 2D displacement estimation in ultrasound imaging," EURASIP J. Adv. Signal Process., vol. 2005, no. 8, pp. 1212
1220,2005

[35] S. Salles, H. Liebgott, D. Garcia, and D. Vray, "Full 3-D transverse oscillations: A method for tissue motion estimation," IEEE Trans. Ultrason., Ferroelec., Freq. Contr., vol. 62, no. 8, pp. 1473-1485, 2015.

[36] C. Kasai, K. Namekawa, A. Koyano, and R. Omoto, "Real-Time TwoDimensional Blood Flow Imaging using an Autocorrelation Technique," IEEE Trans. Son. Ultrason., vol. 32, pp. 458-463, 1985.

[37] J. A. Jensen, A. H. Brandt, and M. B. Nielsen, "Convex array vector velocity imaging using transverse oscillation and its optimization," IEEE Trans. Ultrason., Ferroelec., Freq. Contr., vol. 62, no. 12, pp. 20432053, 2015.

[38] J. A. Jensen, "Field: A program for simulating ultrasound systems," Med. Biol. Eng. Comp., vol. 10th Nordic-Baltic Conference on Biomedical Imaging, Vol. 4, Supplement 1, Part 1, pp. 351-353, 1996.

[39] J. A. Jensen and N. B. Svendsen, "Calculation of pressure fields from arbitrarily shaped, apodized, and excited ultrasound transducers," IEEE Trans. Ultrason., Ferroelec., Freq. Contr., vol. 39, pp. 262-267, 1992.

[40] B. J. Oosterveld, J. M. Thijssen, and W. A. Verhoef, "Texture of Bmode echograms: 3-D Simulations and experiments of the effects of diffraction and scatterer density," Ultrason. Imaging, vol. 7, no. 2, pp. 142-160, Apr. 1985.

[41] J. A. Jensen, H. Holten-Lund, R. T. Nilsson, M. Hansen, U. D. Larsen, R. P. Domsten, B. G. Tomov, M. B. Stuart, S. I. Nikolov, M. J. Pihl, Y. Du, J. H. Rasmussen, and M. F. Rasmussen, "SARUS: A synthetic aperture real-time ultrasound system," IEEE Trans. Ultrason., Ferroelec., Freq. Contr, vol. 60, no. 9, pp. 1838-1852, 2013.

[42] J. A. Jensen, M. F. Rasmussen, M. J. Pihl, S. Holbek, C. A. VillagomezHoyos, D. P. Bradway, M. B. Stuart, and B. G. Tomov, "Safety assessment of advanced imaging sequences, I: Measurements," IEEE Trans. Ultrason., Ferroelec., Freq. Contr., vol. 63, no. 1, pp. 110-119, 2016.

[43] J. A. Jensen, Estimation of Blood Velocities Using Ultrasound: A Signal Processing Approach. New York: Cambridge University Press, 1996. 